\title{
Integrin alpha 11 in the regulation of the myofibroblast phenotype: implications for fibrotic diseases
}

\author{
Ruchi Bansal $^{1}$, Shigeki Nakagawa ${ }^{2,3}$, Saleh Yazdani ${ }^{1}$, Joop van Baarlen ${ }^{4}$, Anu Venkatesh ${ }^{2,3}$, Anna P Koh ${ }^{2,3}$, \\ Won-Min Song ${ }^{2,3}$, Nicolas Goossens ${ }^{2,3}$, Hideo Watanabe ${ }^{5}$, Mary B Beasley ${ }^{3,6}$, Charles A Powell ${ }^{7}$, Gert Storm ${ }^{1,8}$, \\ Naftali Kaminski ${ }^{9}$, Harry van Goor ${ }^{10}$, Scott L Friedman ${ }^{2,3}$, Yujin Hoshida ${ }^{2,3}$ and Jai Prakash ${ }^{1,11,12}$
}

Tissue fibrosis, characterized by excessive accumulation of aberrant extracellular matrix (ECM) produced by myofibroblasts, is a growing cause of mortality worldwide. Understanding the factors that induce myofibroblastic differentiation is paramount to prevent or reverse the fibrogenic process. Integrin-mediated interaction between the ECM and cytoskeleton promotes myofibroblast differentiation. In the present study, we explored the significance of integrin alpha 11 (ITGA11), the integrin alpha subunit that selectively binds to type I collagen during tissue fibrosis in the liver, lungs and kidneys. We showed that ITGA11 was co-localized with $\alpha$-smooth muscle actin-positive myofibroblasts and was correlatively induced with increasing fibrogenesis in mouse models and human fibrotic organs. Furthermore, transcriptome and protein expression analysis revealed that ITGA11 knockdown in hepatic stellate cells (liver-specific myofibroblasts) markedly reduced transforming growth factor $\beta$-induced differentiation and fibrotic parameters. Moreover, ITGA11 knockdown dramatically altered the myofibroblast phenotype, as indicated by the loss of protrusions, attenuated adhesion and migration, and impaired contractility of collagen I matrices. Furthermore, we demonstrated that ITGA11 was regulated by the hedgehog signaling pathway, and inhibition of the hedgehog pathway reduced ITGA11 expression and fibrotic parameters in human hepatic stellate cells in vitro, in liver fibrosis mouse model in vivo and in human liver slices ex vivo. Therefore, we speculated that ITGA11 might be involved in fibrogenic signaling and might act downstream of the hedgehog signaling pathway. These findings highlight the significance of the ITGA11 receptor as a highly promising therapeutic target in organ fibrosis.

Experimental \& Molecular Medicine (2017) 49, e396; doi:10.1038/emm.2017.213; published online 17 November 2017

\section{INTRODUCTION}

Fibrosis or excessive extracellular matrix (ECM) accumulation results in the distortion of tissue architecture and organ dysfunction. Fibrotic diseases are the major cause of increasing morbidity and mortality, contributing to $45 \%$ of deaths worldwide. ${ }^{1}$ Myofibroblasts are the major ECM-producing cells in many pathological conditions, including organ fibrosis and cancer. ${ }^{2-5}$ Myofibroblasts, depending on the etiology and organ, ${ }^{1,6}$ are derived from various cell types, including resident fibroblasts, stellate cells, bone marrow-derived mesenchymal stem cells, epithelial cells via the epithelial-mesenchymal transition ${ }^{7-9}$ and endothelial cells via the endothelial-mesenchymal transition ${ }^{10}$ processes. In addition to ECM production and remodeling, myofibroblasts provide tissue stiffness due to their contractile nature and elicit pro-angiogenic and pro-inflammatory signals in the form of cytokines and growth factors. ${ }^{1,3,6,11,12}$ Myofibroblasts are therefore an attractive and promising cellular target for the diagnosis of fibrotic diseases and potential antifibrotic therapies. ${ }^{13-15}$

Interactions between cells and their extracellular microenvironment are primarily mediated by a family of cell surface receptors known as integrins, which are recognized as key

\footnotetext{
${ }^{1}$ Targeted Therapeutics, Department of Biomaterials Science and Technology, MIRA Institute for Biomedical Technology and Technical Medicine, Faculty of Science and Technology, University of Twente, Enschede, The Netherlands; ${ }^{2}$ Division of Liver Diseases, Department of Medicine, Liver Cancer Program, Tisch Cancer Institute, New York, NY, USA; ${ }^{3}$ Department of Medicine, Icahn School of Medicine at Mount Sinai, New York, NY, USA; ${ }^{4}$ Laboratorium Pathologie Oost-Nederland, Hengelo, The Netherlands; ${ }^{5}$ Department of Medical Oncology, Dana-Farber Cancer Institute, Harvard Medical School, Boston, MA, USA; ${ }^{6}$ Department of Pathology, Icahn School of Medicine at Mount Sinai, New York, NY, USA; ${ }^{7}$ Division of Pulmonary, Critical Care, and Sleep Medicine, Icahn School of Medicine at Mount Sinai, New York, NY, USA; ${ }^{8}$ Department of Pharmaceutics, Utrecht Institute of Pharmaceutical Sciences, Faculty of Science, Utrecht University, Utrecht, The Netherlands; ${ }^{9}$ Yale School of Medicine, Pulmonary, Critical Care and Sleep Medicine, New Haven, CT, USA; ${ }^{10}$ Department of Pathology and Medical Biology, University of Groningen, University Medical Center Groningen, Groningen, The Netherlands; ${ }^{11}$ Department of Oncology-Pathology, Karolinska Institute, Stockholm, Sweden and ${ }^{12}$ ScarTec Therapeutics BV, Enschede, The Netherlands

Correspondence: Dr R Bansal or Dr J Prakash, Targeted Therapeutics, Department of Biomaterials, Science and Technology, MIRA Institute for Biomedical Technology and Technical Medicine, University of Twente, Drienerlolaan 5, Enschede 7522 NB, The Netherlands.

E-mail: R.Bansal@utwente.nl or J.Prakash@utwente.nl

Received 9 December 2016; revised 18 April 2017; accepted 14 May 2017
} 
molecules involved in myofibroblast differentiation. ${ }^{16,17}$ Integrins are heterodimeric transmembrane receptors composed of $\alpha$ and $\beta$ subunits that can combine to form 24 different integrin heterodimers. ${ }^{18}$ Integrins regulate cytoskeletal dynamics, thereby influencing a number of crucial cellular processes, for example, cell adhesion, migration and differentiation. ${ }^{19}$ Integrins also have a key role in the activation of growth factors such as transforming growth factor beta (TGF $\beta$ ); for instance, RGD-binding $\alpha \mathrm{v}$ integrins have been shown to be master regulators of TGF $\beta$ activation in different fibrotic models. ${ }^{17,20,21}$ Because integrins have profound effects on fibrosis in multiple organs, it might be, therefore, of utmost importance to understand/elucidate the regulatory roles of integrins and to define the strategies of modulating integrins for the development of effective antifibrotic approaches.

Accumulation of collagens (predominantly collagen I) in liver fibrosis and other fibrotic tissues is a common characteristic in fibrotic diseases. Among four collagen receptors, $\alpha 1 \beta 1, \alpha 2 \beta 1, \alpha 10 \beta 1$ and $\alpha 11 \beta 1,{ }^{22}$ integrin $\alpha 11 \beta 1$ preferentially binds to type I collagen ${ }^{23}$ and has been shown to be expressed on cultured embryonic fibroblasts ${ }^{24}$ and cardiac fibroblasts. ${ }^{25} \alpha 11 \beta 1$ has been shown to be upregulated by TGF $\beta^{26,27}$ and regulates embryonic mesenchymal cell differentiation on the collagen matrix. ${ }^{25,27,28}$ More recently, the role of integrin $\alpha 11 \beta 1$ was implicated in inducing tumor growth and the metastatic potential of small-cell lung carcinoma cells. $^{29}$ However, the functional significance of integrin alpha 11 (ITGA11), its cellular distribution and contribution to fibrotic diseases remains largely undefined.

In this study, we examined the expression and localization of ITGA11 in human organ fibrosis, including liver cirrhosis, renal fibrosis and lung fibrosis, and have assessed the significance of ITGA11 during TGF $\beta$-induced transdifferentiation of hepatic stellate cells to myofibroblasts. Furthermore, we investigated the signaling pathway that regulates ITGA11 expression and liver fibrogenesis.

\section{MATERIALS AND METHODS}

\section{Human tissues}

Human liver specimens were obtained from the autopsy of patients with liver cirrhosis $(n=5)$ anonymously provided by the Laboratory Pathology Netherlands (LabPON) and Mount Sinai Hospital, New York, USA. Normal liver tissue $(n=4)$ was collected from patients receiving hepatic resections for non-tumoral diseases, including hepatic adenoma and focal nodular hyperplasia. Nephrectomy specimens $(n=4)$ used in this study were obtained from renal transplant recipients with chronic transplant dysfunction and were anonymously provided by the Department of Internal Medicine, Division of Nephrology, University Medical Center Groningen, Groningen, The Netherlands. Fibrotic $(n=5)$ and non-fibrotic $(n=5)$ lung tissues were collected at Mount Sinai Hospital from the patients with lung disease, including pneumothorax and lung cancer. Upon Institutional Review Board approval and after written informed consent from patients, the tissue specimens were collected. The use of human tissues was approved by the respective Local Medical Ethics Committee, and the experimental protocols were performed in accordance with institutional guidelines and regulations.

\section{Cell lines}

Primary Human hepatic stellate cells (HSCs) were purchased from Zen-Bio (Durham, NC, USA) and were grown in stellate cell growth medium SteCM (ScienCell, Carlsbad, CA, USA) according to the manufacturer's instructions. HepG2 and THP1 cells were purchased from ATCC (Manassas, VA, USA) and ECACC (Sigma, St Louis, MO, USA), respectively, and were cultured in Dulbecco's modified Eagle's medium and RPMI-1640 medium (Lonza, Verviers, Belgium), supplemented with $2 \mathrm{~mm}$ L-glutamine, $10 \%$ fetal bovine serum (FBS, Lonza) and antibiotics $\left(50 \mathrm{U} \mathrm{ml}^{-1}\right.$ Penicillin and $50 \mu \mathrm{g} \mathrm{ml}^{-1}$ streptomycin, Sigma) respectively. No cell line used in this paper is listed in the database of commonly misidentified cell lines that is maintained by the International Cell Line Authentication Committee (http://iclac.org/databases/cross-contaminations/). All cell lines were tested negative for mycoplasma contamination.

\section{Stable ITGA11 knockdown in HSCs}

To generate stable ITGA11 knockdown (ITGA11-KD) cells, primary HSCs were transfected with lentiviral shRNA ITGA11 plasmids (Santa Cruz Biotechnology, Dallas, TX, USA) using Lipofectamine 2000 (Invitrogen, Carlsbad, CA, USA) as per the manufacturer's instructions. Control cells were transfected with control/scrambled shRNA plasmid. After $24 \mathrm{~h}$ of transfection, stably transfected cells were selected by $2 \mu \mathrm{g} \mathrm{ml}^{-1}$ puromycin (Invitrogen) for 6-8 weeks. ITGA11$\mathrm{KD}$ and control cells were evaluated for the effects on contractility, migration/wound healing and several fibrotic parameters using staining and quantitative PCR (qPCR).

\section{D collagen-I gel contraction assay}

A collagen suspension $(5 \mathrm{ml})$ containing $3.0 \mathrm{ml}$ of collagen G1 ( $5 \mathrm{mg} \mathrm{ml}^{-1}$, Matrix Biosciences, Morlenbach, Germany), $0.5 \mathrm{ml}$ of $10 \times$ M199 medium (Sigma), $85 \mu$ of $1 \mathrm{~N} \mathrm{NaOH}$ (Sigma) and sterile water was mixed with $1.0 \mathrm{ml}\left(2 \times 10^{6}\right)$ of cells. The collagen gel and cell suspension $(0.6 \mathrm{ml}$ per well) was plated in a 24 -well culture plate and was allowed to polymerize for $1 \mathrm{~h}$ at $37^{\circ} \mathrm{C}$. For the effect studies, polymerized gel was incubated with $1 \mathrm{ml}$ of $0.5 \%$ FBS-containing medium with or without human recombinant $\operatorname{TGF} \beta\left(5 \mathrm{ng} \mathrm{ml}^{-1}\right)$ (Peprotech, Rocky Hill, NJ, USA) together with $10 \mu \mathrm{M}$ LDE225 (Erismodegib, Selleckchem, Boston, NY, USA) followed by detachment of the gels from the culture wells. For other experiments, medium with or without TGF $\beta$ was added before gel detachment. Photographs were made with a digital camera at different time points $(0,24,48$ and $72 \mathrm{~h})$. The size of the gels was digitally measured and normalized with their respective well size in each image. Gel contraction experiments were performed in duplicate in three independent experiments.

\section{Wound-healing assay}

Cells were plated in 12-well culture plates $\left(1 \times 10^{5}\right.$ cells per well) for $24 \mathrm{~h}$ and were starved overnight in $0.5 \%$ FBS-containing medium. A standardized scratch was made using a $200-\mu$ l pipette tip fixed in a holder. Cells were then washed twice and were incubated with and without $5 \mathrm{ng} \mathrm{ml}^{-1} \mathrm{TGF} \beta$. To measure the migratory response of the cells into the scrape wounds, microscopic photographs were taken at 0 and $24 \mathrm{~h}$. Images were analyzed using NIH ImageJ software (NIH, Bethesda, MD, USA) to calculate the area of the scratch wound and were represented as the percentage of wound healed relative to the control wells. Wound-healing experiments were performed in duplicate in three independent experiments. 
Alamar blue assay

To assess the effects on proliferation, cells (control HSCs and ITGA11KD HSCs) plated in 96-well plates were serum starved for $24 \mathrm{~h}$ and were then incubated with fresh medium with or without TGF $\beta$ $\left(5 \mathrm{ng} \mathrm{ml}^{-1}\right)$ for 24 and $48 \mathrm{~h}$. At the indicated time points, cells were incubated with Alamar Blue reagent (Invitrogen) for $4 \mathrm{~h}$. The results
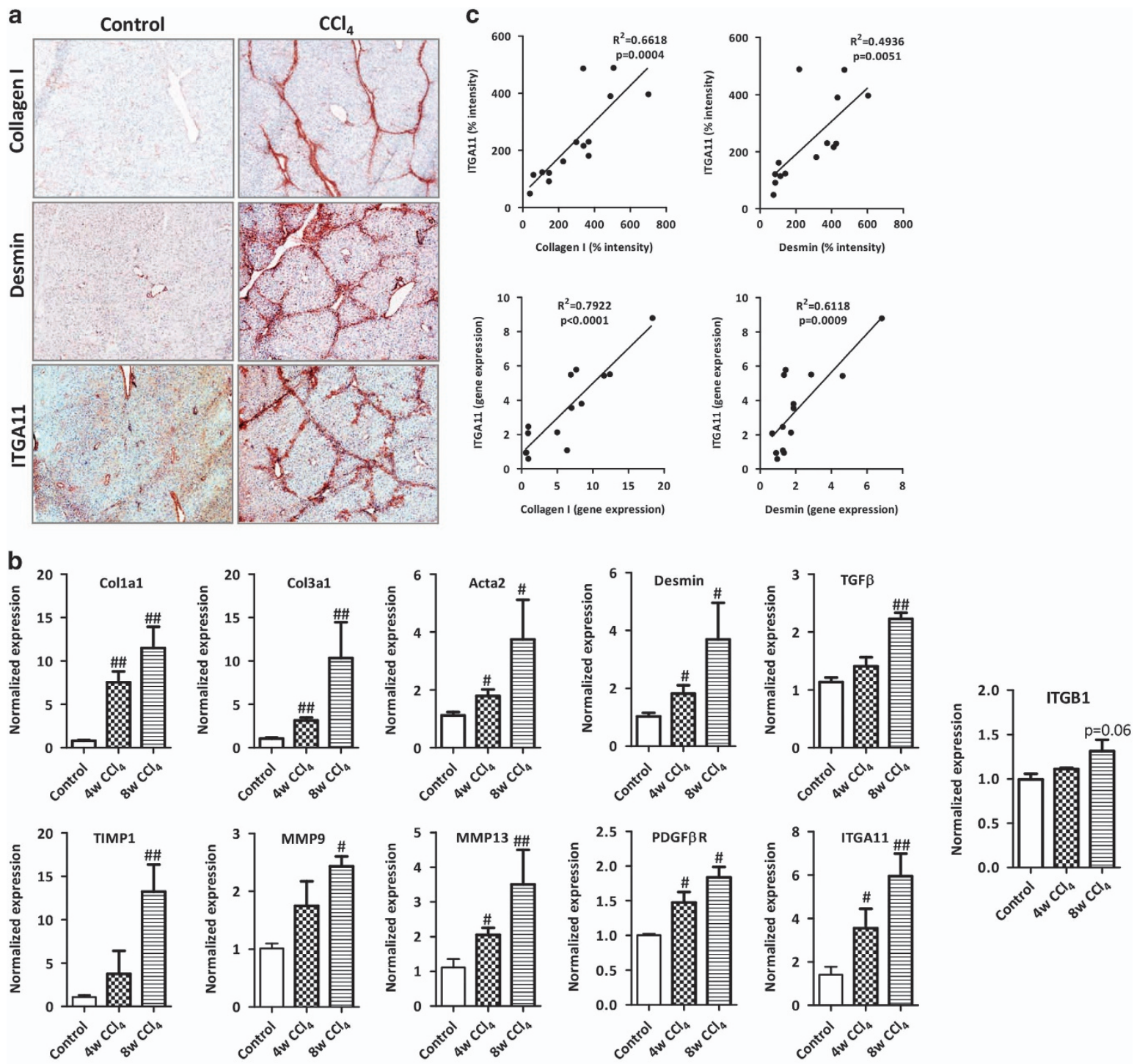

d
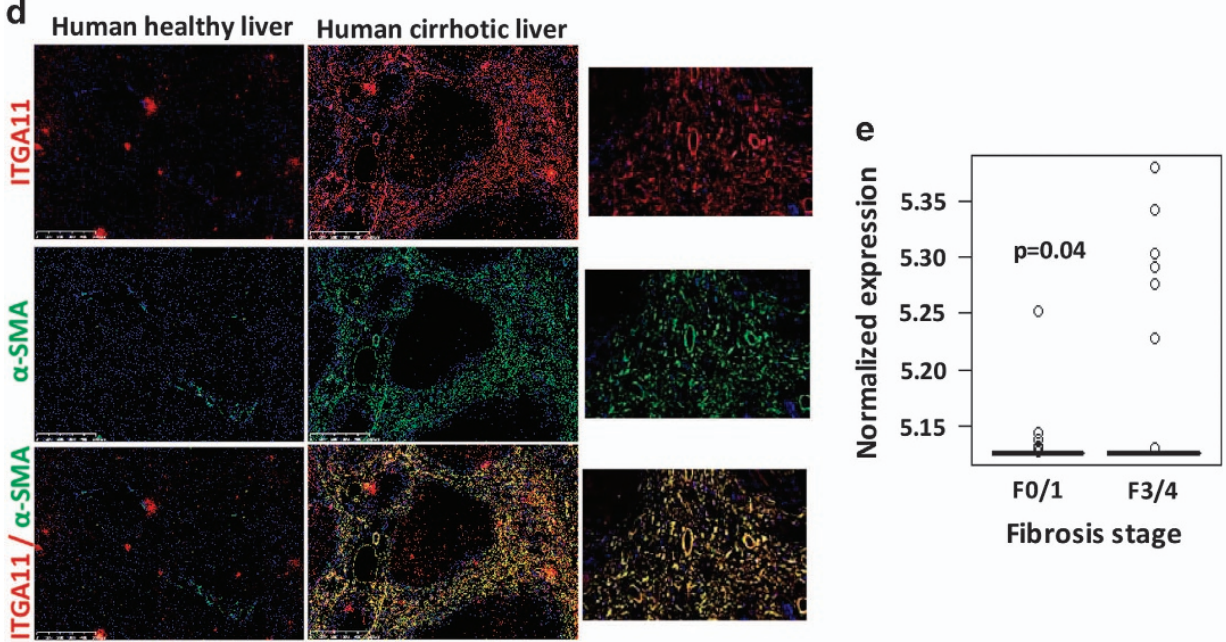
are represented as the percentage of cell viability. All measurements were performed in duplicate in four independent experiments.

\section{Hedgehog parameters and the effect of hedgehog ligand on human control HSCs and ITGA11-KD HSCs}

Cells were seeded in 12 -well plates $\left(8 \times 10^{4}\right.$ cells per well $)$ and were cultured overnight. Cells were serum-starved for $24 \mathrm{~h}$ and then were incubated with $5 \mu \mathrm{g} \mathrm{ml}^{-1}$ of Shh ligand (Peprotech) for $24 \mathrm{~h}$. Cells were lysed with RNA lysis buffer to perform quantitative real-time PCR analysis for hedgehog signaling pathway parameters and ITGA11 expression. qPCR analysis was performed in at least three independent experiments.

\section{In vitro effects of Hedgehog inhibitor in human HSCs}

Cells were seeded in 24 -well plates $\left(3 \times 10^{4}\right.$ cells per well for staining) and 12 -well plates $\left(8 \times 10^{4}\right.$ cells per well for qPCR analysis) and were cultured overnight. To assess the effects on fibrotic parameters, cells were serum-starved for $24 \mathrm{~h}$ and then were incubated with starvation medium alone, $10 \mu \mathrm{M}$ LDE225 (Erismodegib) and $5 \mathrm{ng} \mathrm{ml}^{-1} \mathrm{TGF} \beta 1$ for $24 \mathrm{~h}$. Cells (24-well plates) were then fixed with chilled acetone: methanol (1:1) for $20 \mathrm{~min}$, dried and stained for collagen-I, alpha smooth muscle actin ( $\alpha$-SMA) and vimentin (refer to Supplementary Table 1). In addition, cells (12-well plates) were lysed with RNA lysis buffer to perform quantitative real-time PCR analyses. Staining and qPCR analysis was performed in at least three independent experiments.

\section{Atomic force microscopy (AFM) \\ All AFM measurements were carried out using a Bioscope Catalyst AFM with a Nanoscope V controller (Bruker, Santa Barbara, CA, USA) mounted on an inverted microscope (Axiovert 200, Carl Zeiss, Heidelberg, Germany). The AFM instrument was equipped with a $150 \times 150 \mu \mathrm{m}^{2}$ scanner with a vertical range of $25 \mu \mathrm{m}$. The optical microscope was used to select the desired cell and to position the AFM tip. A rectangular silicon nitride cantilever (NSC36, type C, MikroMasch, Wetzlar, Germany) with a force constant of $\sim 0.6 \mathrm{~N} \mathrm{~m}^{-1}$ was used. Fixed cells (fixed using $4 \%$ paraformaldehyde) were imaged with $512 \times 512$ pixels $^{2}$ in the contact mode in air at a scan rate of $1 \mathrm{~Hz}$. Multiple scans were acquired to image the whole cell. At least 4-5 cells per condition were imaged to obtain representative images. All the AFM images were processed by the Nanoscope Analysis software (Bruker).}

\section{Ex vivo organotypic culture of clinical liver tissue}

De-identified liver tissues were obtained from liver surgeries performed at Mount Sinai Medical Center, and anonymous use was approved by the institutional review board. Fresh liver tissues were sliced into 300- $\mu$ m-thick tissue sections using the Krumdieck Tissue Slicer MD6000 (Alabama Research and Development, Munford, AL, USA) and were cultured with Williams' medium E supplemented with $10 \%$ FBS, $100 \mathrm{U} \mathrm{ml}^{-1}$ penicillin, $100 \mu \mathrm{g} \mathrm{ml}^{-1}$ streptomycin and $2.5 \mu \mathrm{g} \mathrm{ml}^{-1}$ amphotericin B on a heated shaker $\left(37^{\circ} \mathrm{C}\right)$ with medium alone, $10 \mu \mathrm{M}$ or $15 \mu \mathrm{M}$ LDE225 for $48 \mathrm{~h}$ as described earlier. ${ }^{30}$ Harvested tissues were snap-frozen for RNA extraction. ${ }^{31}$ These experiments were performed in three patients $(n=3)$ with three liver slices per patient individually treated and combined for further analysis.

\section{Animal experiments}

All the animal experiments in this study were performed in strict accordance with the guidelines and ethical regulations for the Care and Use of Laboratory Animals, Utrecht University, The Netherlands. The protocols were approved by the Institutional Animal Ethics Committee of the University of Twente, The Netherlands.

\section{$\mathrm{CCl}_{4}$-induced liver fibrosis mouse model}

Male C57BL/6 mice (8-10-week old; $n=5$ per group) were treated with intraperitoneal injections with olive oil or carbon tetrachloride $\left(\mathrm{CCl}_{4}, 1 \mathrm{ml} \mathrm{kg}{ }^{-1}\right.$ prepared in olive oil) twice weekly for 8 weeks and killed, and then the livers and other organs were collected for subsequent analysis.

\section{$\mathrm{CCl}_{4}$-induced acute liver injury mouse model}

Male C57BL/6 mice were treated with a single intraperitoneal injection of olive oil or $\mathrm{CCl}_{4}\left(1 \mathrm{ml} \mathrm{kg}^{-1}\right.$ in olive oil $)$ at day 1 . At days 2 and 3 , $\mathrm{CCl}_{4}$-treated mice received subcutaneous administration of $10 \mathrm{mg} \mathrm{kg}^{-1}$ Hedgehog inhibitor LDE225 prepared in 1\% dimethyl sulfoxide and $5 \% \quad \beta$-hydroxycyclodextrin or vehicle treatment ( $1 \%$ dimethyl sulfoxide/5\% $\beta$-hydroxycyclodextrin/phosphatebuffered saline) ( $n=5$ per group). At day 4 , all mice were killed, and the livers were harvested.

\section{UUO kidney fibrosis mouse model}

Male $\mathrm{C} 57 \mathrm{Bl} / 6$ mice ( $8-10$ weeks old, $n=5$ per group) were subjected to unilateral ureteral obstruction (UUO) by double ligation of the left ureter proximal to the kidney. Right kidneys were used as contralateral controls. Kidneys were harvested 3 and 7 days after surgery.

\section{Immunohistochemistry and immunofluorescence}

Liver tissues were harvested and transferred to Tissue-Tek OCT embedding medium (Sakura Finetek, Torrance, CA, USA) and snap-frozen in 2-methyl butane chilled in dry ice. Cryosections $(4 \mu \mathrm{m})$ were cut using a Leica CM 3050 cryostat (Leica Microsystems,

Figure 1 Upregulation of ITGA11 in $\mathrm{CCl}_{4}$-induced chronic liver fibrosis in mice and fibrotic human livers. (a) Collagen-I, Desmin and ITGA11-stained liver sections from olive-oil-treated (control) and $\mathrm{CCl}_{4}$-treated (8 weeks, fibrotic) mice. $n=5$ per group. (b) Gene expression of fibrotic parameters (Colla1, Col3a1, Tgf, , Timp1, Mmp9 and Mmp13), myofibroblast activation markers (Acta2, Desmin and Pdgf $\beta$ r) and Itgal1 and Itgb1 in the livers of olive-oil-treated non-fibrotic control mice and $\mathrm{CCl}_{4}$-treated fibrotic mice $(4$ and 8 weeks). $n=5$ per group. ${ }^{\#} P<0.05$ and ${ }^{\# \#} P<0.01$ versus control. (c) Correlative analysis of ITGA11 protein and gene expression with respect to the expression of fibrotic parameters (collagen-I and Desmin). The correlations were assessed using Pearson's correlative analysis. ' $R^{2}$ ' denotes Pearson's correlation coefficient, and ' $P$ ' denotes statistical significance. (d) $\alpha$-SMA- and ITGA11-stained human liver sections ( $n=4)$. Left, healthy human liver; middle, cirrhotic human liver; right, magnified image. Upper, ITGA11 (red); middle, $\alpha$-SMA (green); bottom, merged image. Nuclei are stained blue with DAPI. (e) ITGA11 mRNA expression levels from publicly available transcriptome profiling data sets (www.ncbi.nlm.nih.gov/geo). Liver tissues affected with non-alcoholic fatty liver disease (NAFLD) stratified according to fibrosis stage: stage FO or F1, mild fibrosis $(n=40)$; stage F3 or F4, severe fibrosis $(n=32)$ (GEO accession number: GSE49541). 
Nussloch, Germany). The sections were air-dried and fixed with acetone for $10 \mathrm{~min}$. Cells or tissue sections were rehydrated with phosphate-buffered saline and were incubated with the primary antibody (refer to Supplementary Table 1 ) for $1 \mathrm{~h}$ at room temperature. Cells or sections were then incubated with horseradish peroxidase-conjugated secondary antibody for $1 \mathrm{~h}$ at room temperature. Next, the samples were incubated with horseradish peroxidaseconjugated tertiary antibody or donkey anti-goat Alexa 594-labeled tertiary antibody (Life Technologies, Gaithersburg, MD, USA) for $1 \mathrm{~h}$, followed by washing three times with $1 \times$ phosphate-buffered saline. Thereafter, peroxidase activity was developed using the AEC (3-amino-9-ethyl carbazole) Substrate Kit (Life Technologies) for $20 \mathrm{~min}$, and nuclei were counterstained with hematoxylin (Fluka Chemie, Buchs, Switzerland). For tissue sections, endogenous peroxidase activity was blocked by $3 \% \mathrm{H}_{2} \mathrm{O}_{2}$ prepared in methanol. Cells or sections were mounted with Aquatex mounting medium (Merck, Darmstadt, Germany). The staining was visualized, and the images were captured using light microscopy (Nikon eclipse E600 microscope, Nikon, Tokyo, Japan). For immunofluorescence, sections were mounted with DAPI (4,6-diamidino-2-phenylindole)-containing mounting medium (Sigma) and were examined using the Hamamatsu NanoZoomer Digital slide scanner 2.0HT (Hamamatsu Photonics, Bridgewater, NJ, USA). Cells were also stained with Phalloidin (Life Technologies; 1:200 dilution) for $1 \mathrm{~h}$ to visualize F-Actin filaments.

\section{Western blotting analysis}

Cells were lysed in RIPA buffer (Pierce Thermoscientific, Rockford, IL, USA) containing protease inhibitor cocktail and phosphatase inhibitors (Roche Diagnostics, Mannheim, Germany). The samples were boiled in standard protein sample buffer and were subjected to sodium dodecyl sulfate-polyacrylamide gel electrophoresis with 4-20\% Tris-glycine gels (Life Technologies) followed by protein transfer onto a polyvinylidene difluoride membrane. The membranes were developed according to the standard protocols using primary and secondary antibodies (refer to Supplementary Table 1). The bands were visualized using ECL detection reagent (Perkin Elmer Inc., Waltham, MA, USA) and were photographed using the FluorChem a

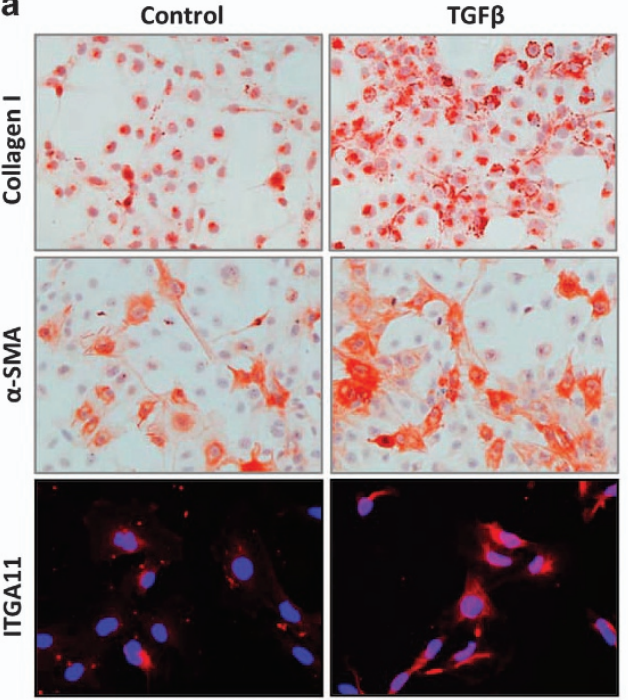

b
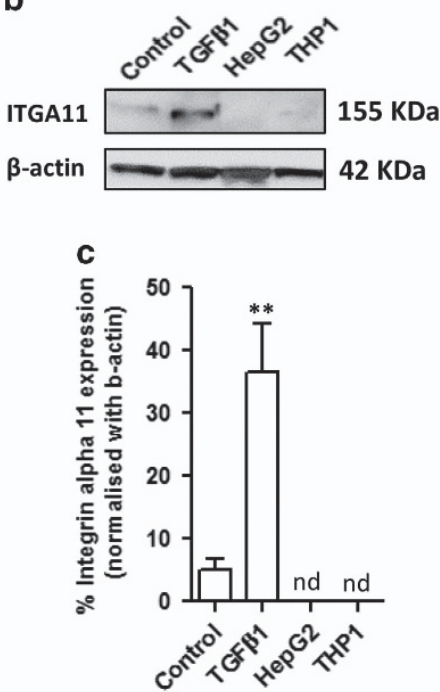
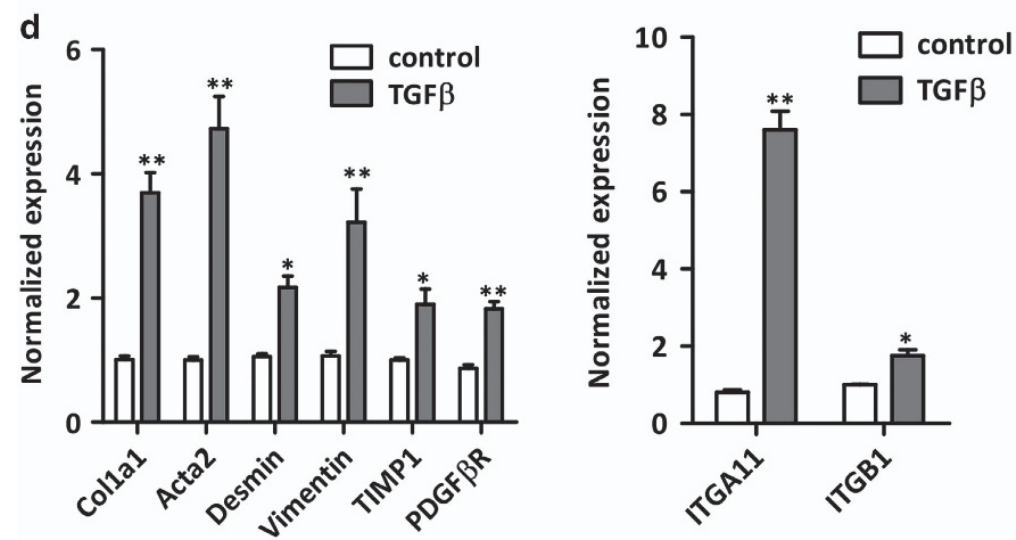

Figure 2 Increased ITGA11 expression in human hepatic stellate cells following TGF $\beta$ treatment. (a) Collagen I-, $\alpha$-SMA- and ITGA11 (ITGA11, red; DAPI, blue)-stained human HSCs treated with medium (control) or TGF $\beta\left(5 \mathrm{ng} \mathrm{ml}^{-1}\right.$ ). (b) Western blotting and (c) analysis of ITGA11 and $\beta$-actin expression in control and TGF $\beta 1$-activated human HSCs, human hepatocytes (HepG2) and human monocytes (THP1). 'nd' denotes not detected. (d) Gene expression of fibrotic parameters (Co/1 $\alpha 1$, Acta2, desmin, vimentin, TIMP1 and PDGFBR) and ITGA11 and ITGBI in control and TGF $\beta$-activated human HSCs, $n=4$. ${ }^{*} P<0.05$ and ${ }^{*} * P<0.01$ versus control HSCs. 
M Imaging System (ProteinSimple, Alpha Innotech, San Leandro, CA, USA).

\section{Quantitative real-time PCR and $\mathrm{RT}^{2}$ profiler PCR array}

Total RNA from cells and liver tissues was isolated using the GenElute Total RNA Miniprep Kit (Sigma) and SV total RNA isolation system (Promega Corporation, Madison, WI, USA), respectively, according to the manufacturers' instructions. The RNA concentration was quantitated using a UV spectrophotometer (NanoDrop Technologies,
Wilmington, DE, USA). Total RNA $(1 \mu \mathrm{g})$ was reverse transcribed using the iScript cDNA Synthesis Kit (Bio-Rad, Hercules, CA, USA). All primers were purchased from Sigma-Genosys (Haverhill, UK). Real-time PCR was performed using the $2 \times$ SensiMix SYBR and Fluorescein Kit (Bioline GmbH, QT615-05, Luckenwalde, Germany), $20 \mathrm{ng}$ of cDNA and pretested gene-specific primer sets (listed in Supplementary Tables 2 and 3). The cycling conditions for the Bio-Rad CFX384 Real-Time PCR detection system were $95^{\circ} \mathrm{C}$ for 10 min, 40 cycles of $95^{\circ} \mathrm{C} / 15 \mathrm{~s}, 58^{\circ} \mathrm{C} / 15 \mathrm{~s}$ and $72^{\circ} \mathrm{C} / 15 \mathrm{~s}$. Finally, cycle
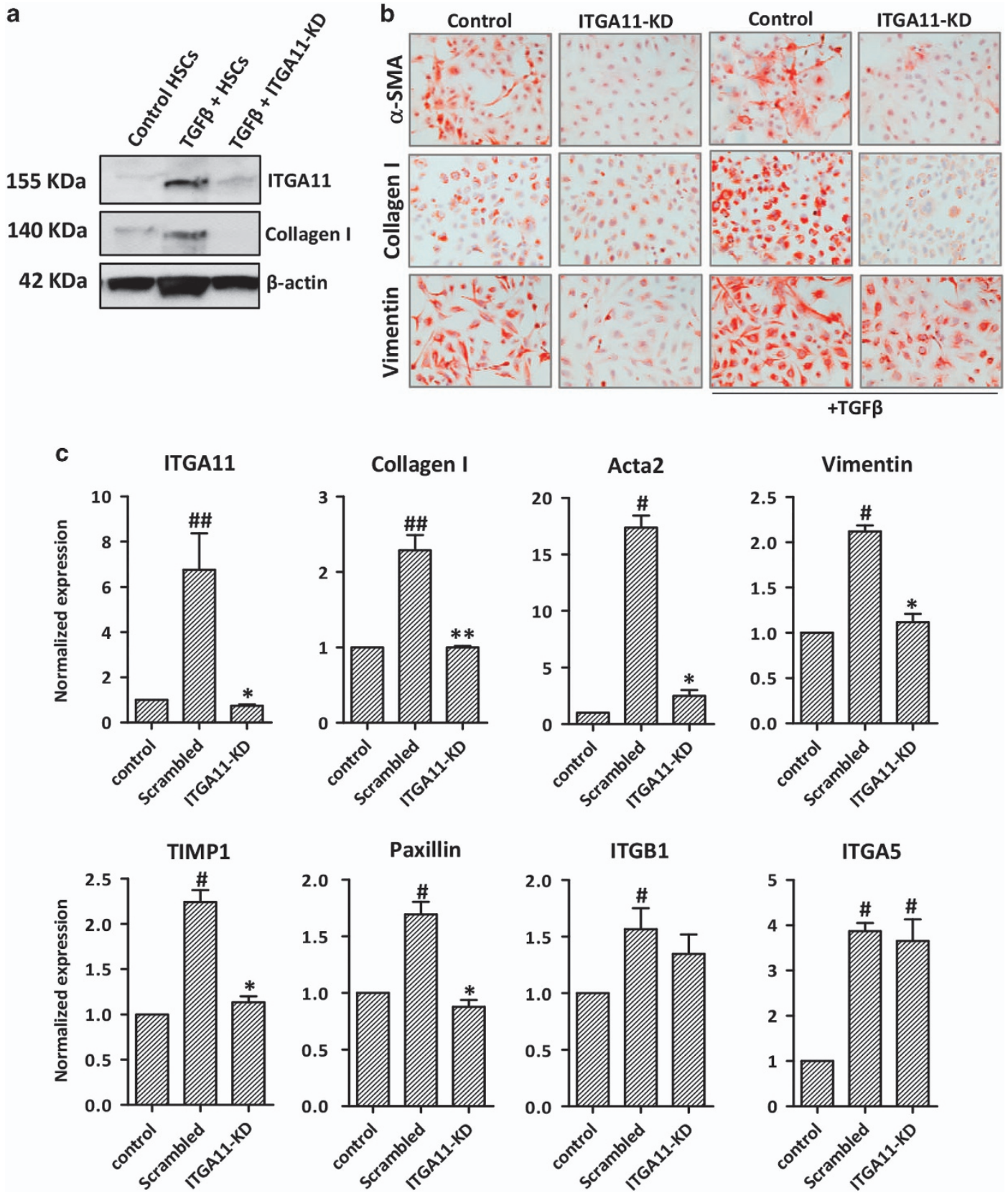

Figure 3 ITGA11 depletion in human HSCs inhibits HSC activation. (a) Western blotting depicting ITGA11, Collagen-I and $\beta$-actin expression in control, TGF $\beta$-treated HSCs and TGF $\beta$-treated ITGA11-KD (ITGA11-knockdown) HSCs. (b) Collagen-I-, $\alpha$-SMA- and vimentinstained control and ITGA11-KD HSCs with or without TGF $\beta$ ( $5 \mathrm{ng} \mathrm{ml}^{-1}$ ). (c) Gene expression of ITGA11, fibrotic parameters (Collagen I, Acta2, vimentin and TIMP1), ECM-adhesion protein (Paxillin), ITGB1 and ITGA5 in control HSCs, TGF $\beta$-treated scrambled HSCs and ITGA11-KD HSCs, $n=4$. ${ }^{\#} P<0.05$ and ${ }^{\# \#} P<0.01$ versus control HSCs. ${ }^{*} P<0.05$ and ${ }^{* *} P<0.01$ versus TGF $\beta$-treated HSCs. Control cells were transfected with control/scrambled shRNA plasmid. ITGA11-KD cells were transfected with the ITGA11 shRNA plasmid. 
threshold $(\mathrm{Ct})$ values were normalized to the reference gene GAPDH (glyceraldehyde 3-phosphate dehydrogenase), and fold changes in expression were calculated using the $2^{-\Delta \Delta C t}$ method. For the $R^{2}$ profiler array, RNA (500 ng) from ITGA11-KD cells and control cells was reverse transcribed using the $\mathrm{RT}^{2}$ First Strand Kit (SABiosciences, Frederick, MD, USA), and qPCR was performed using the human fibrosis PCR array (RT2 Profiler PCR Array PAHS-120Z, SABiosciences) as per the manufacturer's instructions. Six house-keeping genes (ACTB, B2M, GAPDH, HPRT1, RPLP0 and HGDC), RT controls and PCR controls were included in each run. PCR array data were analyzed using the web-based software 'RT ${ }^{2}$ Profiler PCR Array Data Analysis v. 3.5', available at the manufacturer's website.

ITGA11 mRNA expression in the human cohort from the public database

ITGA11 mRNA expression was assessed in the publicly available transcriptome data sets of liver tissue from patients with non-alcoholic steatohepatitis (non-alcoholic fatty liver disease) $(n=72$, GSE49541), renal allograft tissue from patients after allograft renal transplantation ( $n=48$, GSE25902) and lung tissue from patients with or without interstitial lung disease $(n=219$, GSE47460).

\section{Statistical analyses}

The results were expressed as the mean+s.e.m. The graphs and statistical analyses were performed using GraphPad Prism version
5.02 (GraphPad Prism, La Jolla, CA, USA). Analyses were performed using Student's $t$-test (comparison with the control group), while multiple comparisons between different groups were performed using the one-way analysis of variance with the Bonferroni post hoc test. $P<0.05$ was considered significant. Correlations were assessed using Pearson's correlative analysis, and dot plots were generated. For in vitro experiments, no statistical method was used to predetermine the sample size. For in vivo experiments, the sample size was estimated using power analysis-that is, a difference of $20 \%$ with a power of $80 \%(1-\beta)$ and an $\alpha$ of 0.05 . For all in vivo studies, mice were blindly randomized into different groups. The investigators were not blinded to allocation for the in vivo experiments but were blinded to allocation for immunohistochemical analyses. Quantitative data analysis was performed in a blinded manner. No samples that were fully processed for different assays, western blotting or immunohistochemical analysis were excluded. No mice that completed the studies were excluded from the analyses.

\section{RESULTS}

\section{Upregulation of ITGA11 in the fibrotic livers in $\mathrm{CCl}_{4}$ -} induced mouse models

We examined the ITGA11 expression in the mouse fibrotic livers as compared with the healthy livers. Repeated administration of $\mathrm{CCl}_{4}$ for 4 and 8 weeks in mice resulted in extensive bridging fibrosis with a substantial deposition of

Table 1 Genes differentially expressed in ITGA11-KD cells compared with control cells characterized in the Human Fibrosis PCR Array (RT2 ${ }^{2}$ Profiler PCR Array PAHS-0120Z, SABiosciences) and analyzed using the provided RT Profiler PCR Array Data Analysis v. 3.5 software

\begin{tabular}{|c|c|c|c|}
\hline Gene symbol & Refseq & Description & Fold regulatior \\
\hline \multicolumn{4}{|c|}{ Profibrotic and inflammatory cytokines } \\
\hline ACTA2 & NM_001613 & Actin, alpha 2, smooth muscle, aorta & -1.8557 \\
\hline CCL2 (MCP1) & NM_002982 & Chemokine (C-C motif) ligand 2 & -4.2634 \\
\hline IL-1B & NM_000576 & Interleukin 1 , beta & -4.0615 \\
\hline \multicolumn{4}{|c|}{ Extracellular matrix, ECM remodeling and cell adhesion } \\
\hline COL1A2 & NM_000089 & Collagen, type I, alpha 2 & -2.4318 \\
\hline MMP1 & NM_002421 & Matrix metallopeptidase 1 (interstitial collagenase) & -60.6317 \\
\hline MMP3 & NM_002422 & Matrix metallopeptidase 3 (stromelysin 1, progelatinase) & -5.0002 \\
\hline MMP9 & NM_004994 & Matrix metallopeptidase 9 (gelatinase B, 92 kDa gelatinase, 92 kDa type IV collagenase) & -1.948 \\
\hline TIMP1 & NM_003254 & TIMP metallopeptidase inhibitor 1 & -2.0167 \\
\hline TIMP3 & NM_000362 & TIMP metallopeptidase inhibitor 3 & -2.6427 \\
\hline PLAT & NM_000930 & Plasminogen activator, tissue & -1.9079 \\
\hline PLAU & NM_002658 & Plasminogen activator, urokinase & -3.6351 \\
\hline SERPINE1 & NM_000602 & Serpin peptidase inhibitor, clade $\mathrm{E}$ (nexin, plasminogen activator inhibitor type 1 ), member 1 & -2.2377 \\
\hline ITGA2 & NM_002203 & Integrin, alpha 2 (CD49B, alpha 2 subunit of VLA-2 receptor) & -4.8299 \\
\hline \multicolumn{4}{|c|}{ Signal transduction (TGF $\beta$ superfamily and transcription factors) } \\
\hline CEBPB & NM_005194 & CCAAT/enhancer binding protein (C/EBP), beta & -1.9616 \\
\hline STAT1 & NM_007315 & Signal transducer and activator of transcription 1 & -1.8557 \\
\hline TGFBR 1 & NM_004612 & Transforming growth factor, beta receptor 1 & -1.9752 \\
\hline TGFBR2 & NM_003242 & Transforming growth factor, beta receptor 2 & -1.948 \\
\hline TGIF1 & NM_003244 & TGFB-induced factor homeobox 1 & -2.0591 \\
\hline THBS1 & NM_003246 & Thrombospondin 1 & 3.1123 \\
\hline THBS2 & NM_003247 & Thrombospondin 2 & -1.8947 \\
\hline
\end{tabular}

Bold characters indicate genes upregulated or downregulated by $\geqslant 1.8$-fold. 
collagen I and HSC activation (increased desmin expression) compared with olive-oil-treated non-fibrotic control livers (Figure 1a and Supplementary Figure S1). The gene expression of major fibrotic parameters, including Collal (Collagen I), Des (desmin), Acta2 ( $\alpha$-SMA), Col3al (Collagen-III), TGF $\beta$, Timp1 (tissue inhibitor of matrix metalloproteases), Mmp9 and
Mmp13 (matrix metalloproteases 9 and 13) and Pdgf $\beta R$ (platelet-derived growth factor receptor beta), were significantly increased in the $\mathrm{CCl}_{4}$-treated fibrotic livers versus olive-oiltreated control livers (Figure 1b). Importantly, both the protein and gene expression levels of Itga11 were correlatively upregulated with an increasing degree of liver fibrosis (collagen-I and
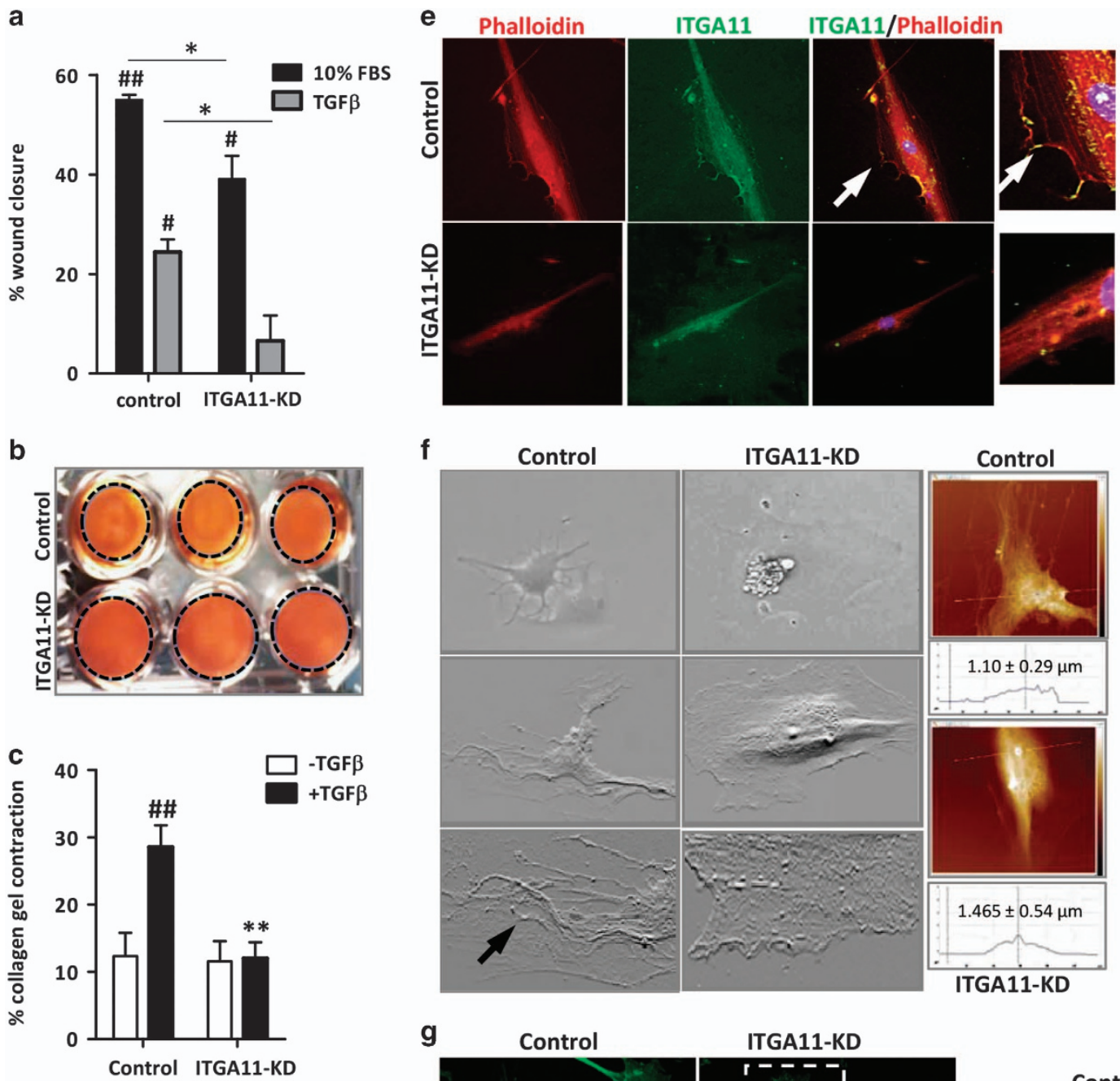

f

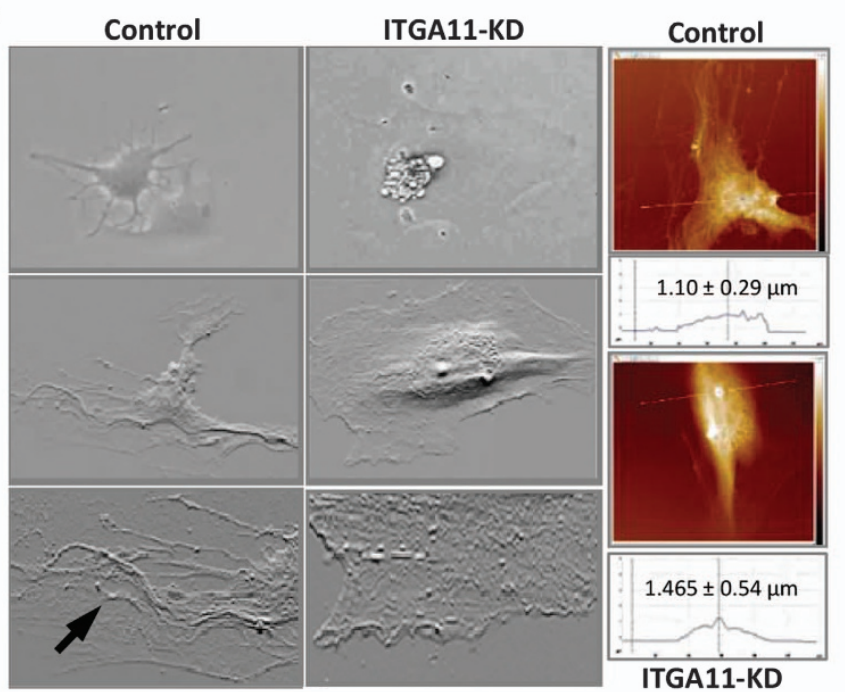

g
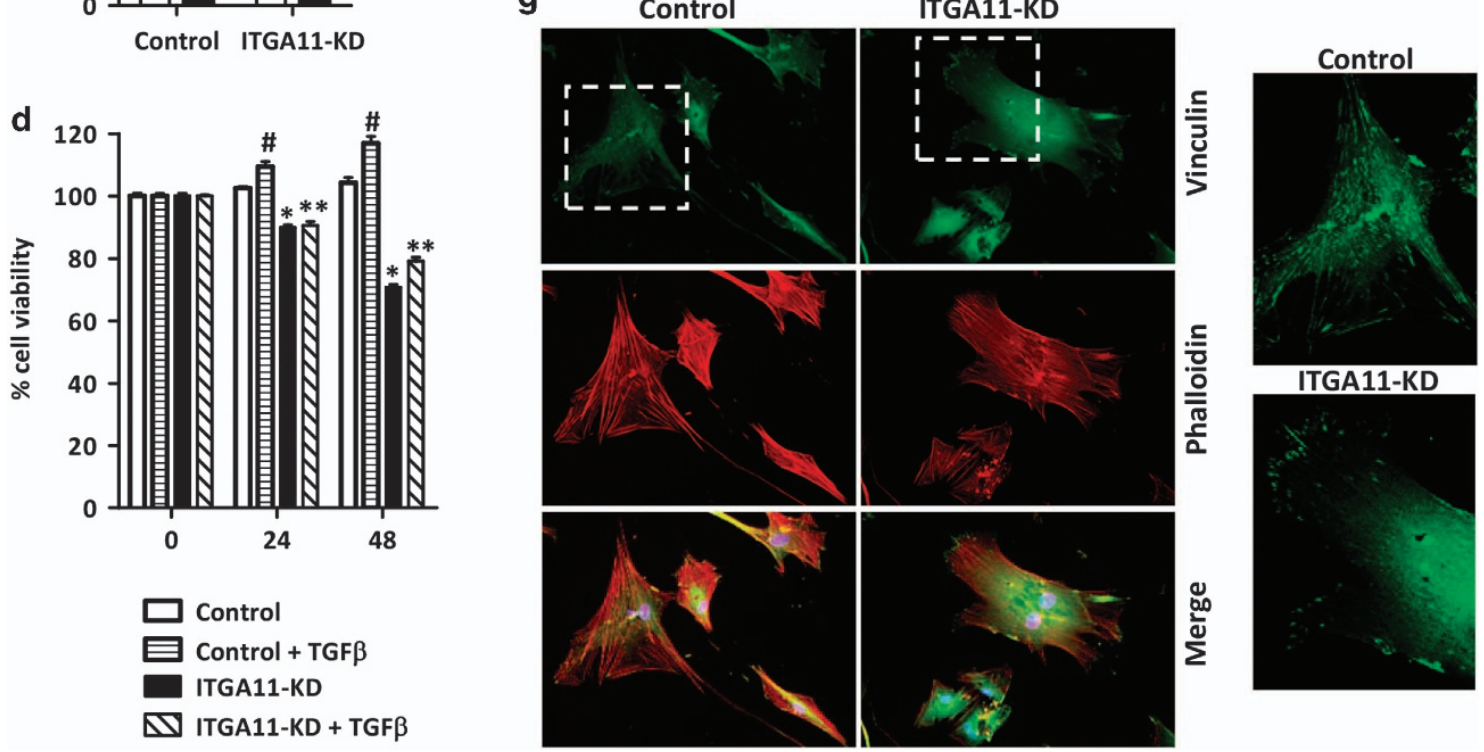
desmin), and the expression was localized in the areas of active fibrogenesis (Figures 1a-c). Because the ITGA11 subunit forms a dimer with the ITGB1 subunit (Integrin beta 1), we also assessed the expression of ITGB1 and observed higher expression of Itgb1 (Integrin beta 1) mRNA expression in 8-weeks $\mathrm{CCl}_{4}$-treated fibrotic livers as compared with the control livers $(P=0.06)$ (Figure $1 \mathrm{~b})$. Additionally, we observed very low expression of ITGA11 in normal healthy livers (Figure 1a) and other normal mouse organs-that is, the heart, kidneys, lungs and spleen-mainly confined to the collagen-I-positive regions (Supplementary Figure S2).

\section{Upregulation and myofibroblast-specific localization of ITGA11 expression in the human fibrotic livers}

In the human cirrhotic livers, ITGA11 was highly overexpressed and specifically co-localized with $\alpha$-SMA-positive HSCs (Figure 1d). Analysis in non-alcoholic fatty liver disease patients showed a significant induction of ITGA11 expression $(P<0.04)$ in the advanced liver fibrosis stages F3 or F4 (severe fibrosis, $n=32$ ) compared with stage F0 or F1 (mild fibrosis, $n=40$ ) (Figure 1e) as determined from transcriptome data analysis (GEO accession number: GSE49541).

\section{Induction of ITGA11 expression following TGF $\beta$-mediated HSC activation}

To establish that ITGA11 is specifically induced in primary human activated HSCs (liver myofibroblasts), we examined its expression in TGF $\beta$-activated HSCs compared with non-activated control HSCs. As shown in Figures $2 a$ and $b$, upon TGF $\beta$-mediated HSC activation, ITGA11 protein expression was significantly increased concomitantly with collagen-I and the myofibroblast-specific marker $\alpha$-SMA. By contrast, ITGA11 expression remained undetectable in human hepatocytes (HepG2) and human monocytes (THP1) (Figures $2 \mathrm{~b}$ and c). Furthermore, mRNA analysis showed marked induction of several genes associated with the myofibroblastic phenotype, including Colla1, Acta2, Desmin, Vimentin, TIMP1 and PDGF $\beta$ R, and ITGA11 and ITGB1 (Figure 2d).

\section{ITGA11 depletion in HSCs attenuates HSC activation and differentiation}

To implicate ITGA11 in HSC activation, we generated human HSCs with stable ITGA11 knockdown (ITGA11-KD) using shRNA-ITGA11 and evaluated whether the loss of ITGA11 affects their phenotype and TGF $\beta$-mediated activation and differentiation. The knockdown of ITGA11 was confirmed using western blotting and qPCR, with 90\% reduction in ITGA11 expression compared with scrambled-shRNAtransfected control HSCs (Figures 3a and c). Significantly, we found that ITGA11-KD HSCs strongly inhibited TGF $\beta$-induced differentiation ( $\alpha$-SMA) and ECM production (collagen-I and vimentin) compared with control (scrambled shRNA) HSCs (Figures 3b and c). ITGA11 knockdown significantly downregulated mRNA transcripts of major fibrotic parameters such as collagen-I, Acta2, Vimentin, TIMP1 and Paxillin (ECM adhesion protein), whereas no effect on ITGB1 and ITGA5 mRNA levels was observed (Figure 3c). Notably, scrambledshRNA-transfected control HSCs demonstrated increased $\alpha$-SMA expression (Figure 3c) compared with control HSCs (non-transfected) as shown in Figure 2d.

We further analyzed ITGA11-KD and control HSCs for the mRNA profiling of 84 fibrosis parameters using the $\mathrm{RT}^{2}$ profiler human fibrosis array. Interestingly, we found the downregulation of the following 19 fibrosis-related genes: (a) pro-fibrotic and inflammatory cytokines, for example, Acta2, MCP1 (or CCL2) and interleukin-1 $\beta$; (b) ECM-remodeling and adhesion genes, for example, Collagen 1A2, MMP1, MMP3, MMP9, TIMP1, TIMP3, PLAT, PLAU, SERPINE1 and ITGA2; and (c) TGF $\beta$ superfamily genes, for example, C/EBP, STAT1, TGFBR1, TGFBR2, TGIF1 and THBS2 (Table 1). These findings suggest that ITGA11 indeed regulates HSC activation at different levels. Notably, ITGA11 depletion inhibited the expression of TGF $\beta$ receptors (TGF $\beta$ R1 and TGF $\beta$ R2) and TGIF1 (TGF $\beta$ inducing factor 1 ), implicating a role of ITGA11 in the negative feedback regulation of the TGF $\beta$-signaling pathway (Table 1).

Figure 4 ITGA11 depletion in HSCs inhibits phenotypic transformation of HSCs. (a) Percentage of wound closure (24 h) by control HSCs and ITGA11-KD HSCs under different conditions, $n=4$. $\# P<0.05$, \#\# $P<0.01$ versus control or ITGA11-KD HSCS; ${ }^{*} P<0.05$. Representative images (b) and quantitative analysis (c) of 3D collagen-I gel contraction containing control versus ITGA11-KD HSCs treated

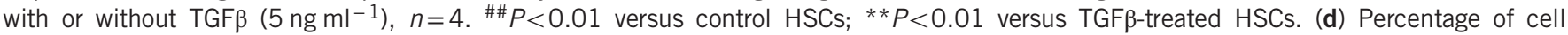
viability of control HSCs and ITGA11-KD HSCs with or without TGF $\beta\left(5 \mathrm{ng} \mathrm{ml}^{-1}\right)$ at different time points $(0,24$ and $48 \mathrm{~h})$ as assessed using the Alamar Blue assay. $n=4$. ${ }^{\#} P<0.05$ versus control; ${ }^{*} P<0.05$ and ${ }^{*} P<0.01$ versus control or TGF $\beta$-treated HSCs. (e) ITGA11KD and control HSCs stained with phalloidin (red, first column), ITGA11 (green, second column); third column, merged image; fourth column, magnified merged image (depicting co-localization), and arrows depict the ITGA11/phalloidin co-stained protrusions. Nuclei are stained blue with DAPI. (f) AFM images depicting the adhesion of stained control HSCs and ITGA11-KD HSCs. The upper panel depicts microscopic images, the middle panel depicts AFM images and the bottom panel depicts the magnified edges. The right panel shows the height of cells (average \pm s.d., $n=4$ ) as measured by AFM. (g) Control (first column) and ITGA11-KD (second column) HSCs stained with vinculin (green, upper panel) and phalloidin (red, middle panel); lower panel, merged image. The third column shows the magnified vinculin-stained images. Nuclei are stained blue with DAPI. Control cells were transfected with control/scrambled shRNA plasmid. ITGA11KD cells were transfected with the ITGA11 shRNA plasmid. 
ITGA11 depletion in HSCs inhibits the phenotypic transformation of HSCs

Because HSCs can migrate to the sites of tissue injury during fibrogenesis and differentiate into contractile myofibroblasts that promote liver stiffness, we examined the effect of ITGA11 knockdown on migration using wound-healing assays and contractility of HSCs using the 3D collagen contraction assay. ITGA11-KD HSCs displayed attenuated migration after $24 \mathrm{~h}$

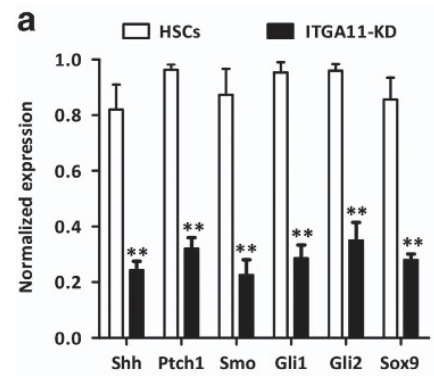

b

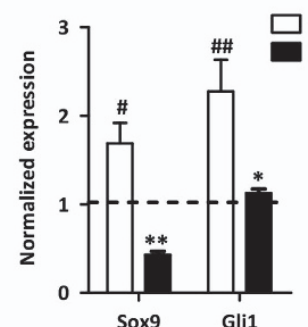

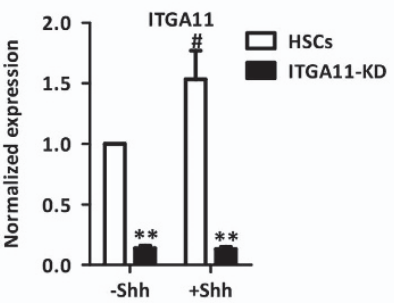

d

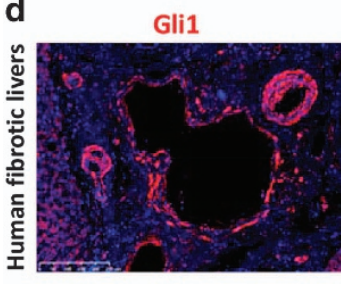

$\alpha-S M A$

Gli1 $+\alpha-S M A$
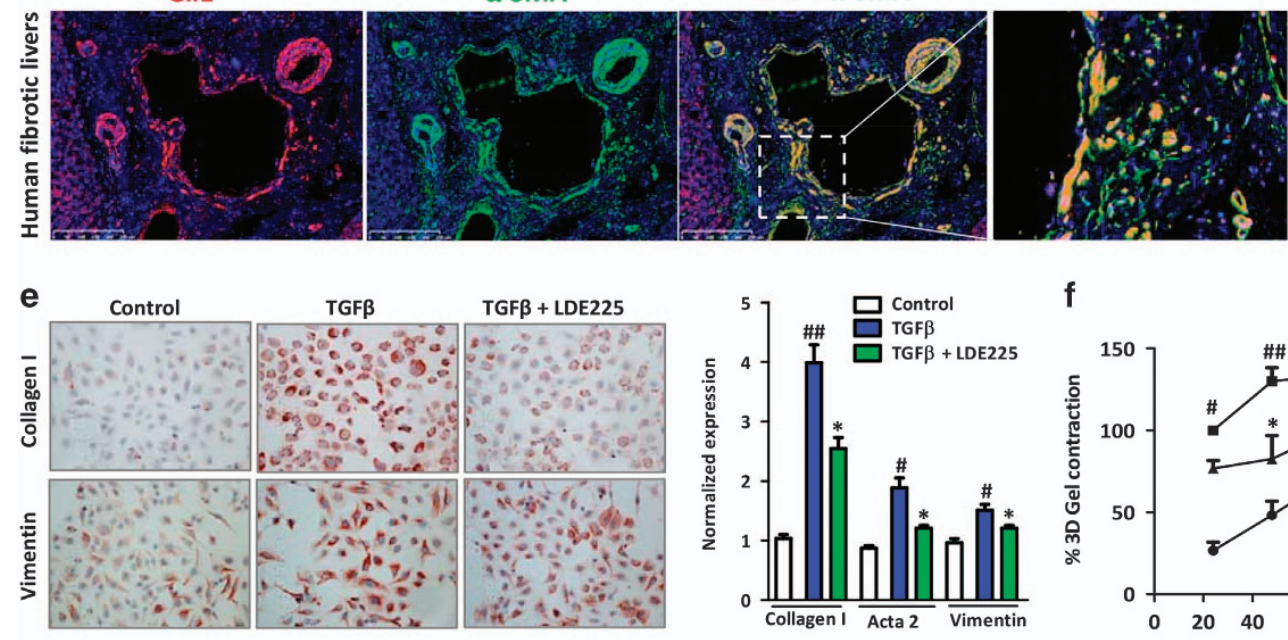

f
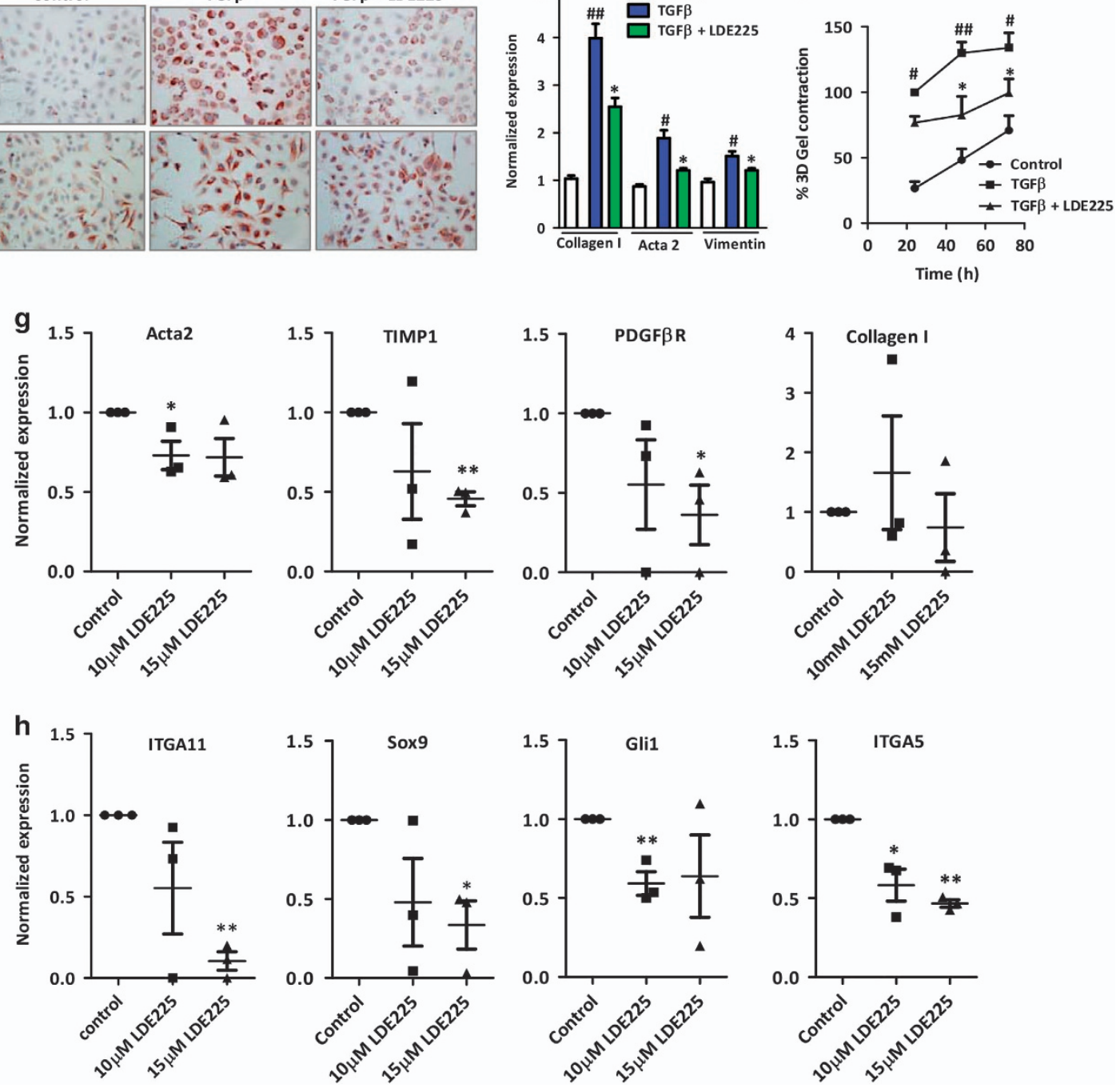
compared with control HSCs upon migratory stimulation with $10 \%$ FBS (approximately $30 \%$ inhibition) or TGF $\beta$ (approximately 60\% inhibition) (Figure $4 \mathrm{a}$ and Supplementary Figure S3). Furthermore, ITGA11-KD HSCs displayed significantly diminished collagen gel contraction upon TGF $\beta$ activation compared with scrambled control HSCs (approximately 60\% inhibition; Figures $4 \mathrm{~b}$ and c). Because these effects can be related to differences in proliferation, we performed the Alamar Blue assay at different incubation times $(0,24$ and $48 \mathrm{~h})$ with or without TGF $\beta$. We observed approximately $10 \%$ inhibition in cell proliferation after $24 \mathrm{~h}$ of TGF $\beta$ incubation and approximately $20 \%$ inhibition in cell proliferation after $48 \mathrm{~h}$ of incubation (Figure $4 \mathrm{~d}$ ). Therefore, the differences observed in wound-healing assays and contraction assays (60\% inhibition) could partially reflect the reduced proliferative activity of ITGA11-KD HSCs (Figures 4a-d).

To further investigate the significance of ITGA11 on the functional characteristics of HSCs, we studied their adhesion to the surface and changes in protrusions. We performed phalloidin/ITGA11 co-immunostaining and found that ITGA11-KD HSCs have significantly reduced actin fibers and focal adhesion points compared with control scrambled HSCs (Figure 4e). To further confirm these changes in adhesion, we performed AFM to visualize the detailed focal adhesions. As shown in Figure 4f, many focal adhesions were present in the scrambled control HSCs as compared with ITGA11-KD HSCs where the cellular edges were blunted, indicating the loss of focal adhesions and poor adhesion to the surface. We also quantified the cell height from the surface because if the cells are tightly adherent, the cells have less height compared with the weakly adhered cells. Interestingly, we observed that the height of the control scrambled HSCs was significantly lower than that of ITGA11-KD HSCs $(1.10 \pm 0.29$ versus $1.465 \pm 0.54 \mu \mathrm{m})$ (Figure 4f), reflecting an important role of ITGA11 in cellular adhesion. In addition, we performed vinculin and phalloidin staining on TGF $\beta$-activated control scrambled HSCs and ITGA11-KD HSCs and observed highly significantly reduced vinculin and phalloidin staining as shown in Figure 4g. Notably, vinculin and phalloidin staining was significantly differently localized in ITGA11-KD cells as compared with control HSCs as depicted in higher magnification images (Figure $4 \mathrm{~g}$ ). These data strongly demonstrate that ITGA11 is a functionally and distinctively active receptor in the regulation of the activation and differentiation of HSCs and mediate the contraction, migration and adhesion of HSCs.

\section{Crosstalk between ITGA11 and the Hedgehog signaling pathway}

Recently, it has been shown that myofibroblasts mainly originate from Gli1+ (glioma-associated oncogene homolog 1) perivascular progenitor cells. ${ }^{32}$ Because Gli1 is a transcription factor of the hedgehog pathway, we investigated the effect of ITGA11-KD on the expression levels of hedgehog pathway-related components- that is, sonic hedgehog ligand (SHH), patched-1 receptor (PTCH1), Smoothened (SMO), a downstream protein in the pathway, and signaling molecules Gli1, Gli2 transcription factors and sex-determining region Y box 9 (Sox9) in HSCs. Interestingly, we found that the mRNA expression levels of hedgehog pathway-related components are highly significantly inhibited following ITGA11 knockdown (Figure 5a). Furthermore, TGF $\beta$-induced expression of Gli1 and Sox9 was significantly inhibited in ITGA11-KD HSCs as compared with control scrambled HSCs (Figure 5b), suggesting that ITGA11 mediates its effect via the hedgehog pathway, or ITGA11 may be involved in the downstream hedgehog signaling pathway and positively regulate Gli1 and Sox9 expression. To study the direct involvement of hedgehog and ITGA11, we incubated control and ITGA11-KD HSCs with $5 \mu \mathrm{g} \mathrm{ml}^{-1}$ of Shh ligand and investigated ITGA11 gene expression. We found control HSCs showed the induction in ITGA11 expression following incubation with Shh ligand compared with ITGA11-KD, further demonstrating that ITGA11 is downstream of the hedgehog signaling pathway and regulates HSC activation and differentiation (Figure 5c). We further examined the expression of Gli1 on $\alpha$-SMA-positive myofibroblasts and confirmed the perivascular co-localization of Gli1 and $\alpha$-SMA (Figure $5 \mathrm{~d}$ ), corroborating the findings of a recent study. ${ }^{32}$

Figure 5 Myofibroblastic localization of Gli1 and the effect of hedgehog inhibition on TGF $\beta$-activated human HSCs in vitro and human liver slices ex vivo. (a) Gene expression of hedgehog pathway-related genes (Shh, Ptch1, Smo, Gli1, Gli2 and Sox9) in ITGA11-KD HSCs versus control HSCs, $n=3$. ${ }^{* *} P<0.01$ represents significance versus control HSCs. (b) Gene expression of hedgehog pathway-related genes (Sox9 and Gli1) in TGF $\beta$-treated ITGA11-KD HSCs versus TGF $\beta$-treated control HSCs, $n=4$. \# $P<0.05$ and \#\# $P<0.01$ versus control HSCs (dashed line); ${ }^{*} P<0.05$ and ${ }^{* *} P<0.01$ versus TGF $\beta$-treated HSCs. (c) Gene expression of ITGA11 in control HSCs and ITGA11-KD HSCs with or without Shh $\left(5 \mu \mathrm{g} \mathrm{ml}{ }^{-1}\right), n=3$. ${ }^{\#} P<0.01$ versus Shh-treated HSCs, ${ }^{*} P<0.01$ versus control HSCs. (d) $\alpha$-SMA and Gli1 coimmuno-stained human liver sections. Gli1 (red, first column), $\alpha$-SMA (green, second column) and merged image (third column). Fourth column shows the magnified image depicting co-localization. Nuclei are stained blue with DAPI. (e) Collagen-I- and vimentin-stained HSCs treated with or without TGF $\beta\left(5 \mathrm{ng} \mathrm{ml}^{-1}\right) \pm 10 \mu \mathrm{m}$ of the hedgehog inhibitor (LDE225). Gene expression of fibrotic parameters collagen-I, $\alpha$ SMA and vimentin in HSCs treated with medium alone, TGF $\beta\left(5 \mathrm{ng} \mathrm{ml}^{-1}\right) \pm 10 \mu \mathrm{m} \operatorname{LDE} 225, n=3$. ${ }^{\prime} P<0.05$, \#\# $P<0.01$ versus the control cells; ${ }^{*} P<0.05$ versus TGF $\beta$-treated cells. (f) Graph depicts \% 3D collagen-I gel contraction after 24,48 and $72 \mathrm{~h}$ of treatment with or without TGF $\beta\left(5 \mathrm{ng} \mathrm{ml}^{-1}\right) \pm 10 \mu \mathrm{m}$ LDE225, $n=3$. ${ }^{\#} P<0.05$, \#\# $P<0.01$ versus control cells; ${ }^{*} P<0.05$ versus TGF $\beta$-treated cells. Gene expression of Acta2, TIMP1, PDGF $\beta$ R and Collagen-I (g) and ITGA11, Sox9, Gli1 and ITGA5 (h) in the slices obtained from fibrotic livers from human patients incubated with medium (control) or 10 or $15 \mu \mathrm{m}$ LDE225. $n=3$ patients (3 slices each). ${ }^{*} P<0.05$ and ${ }^{* *} P<0.01$ versus control group. Control cells were transfected with control/scrambled shRNA plasmid. ITGA11-KD cells were transfected with the ITGA11 shRNA plasmid. 
Hedgehog pathway inhibition attenuates fibrotic parameters in human HSCs in vitro and human liver slices ex vivo

To further understand the mechanism involved in ITGA11-mediated regulation of myofibroblast differentiation, we examined the effect of a selective hedgehog signaling pathway inhibitor, LDE225 (or Erismodegib), on human HSC activation in vitro in human HSCs and ex vivo in human liver slices. Treatment of HSCs with LDE225 led to a significant reduction in TGF $\beta$-induced $\alpha$-SMA, collagen I and vimentin gene and protein expression (Figure 5e and Supplementary Figure S4). Because ITGA11-KD cells had an attenuated capacity of collagen production, we examined the effect of hedgehog inhibition on HSC contraction and found that LDE225 $(10 \mu \mathrm{M})$ significantly reduced TGF $\beta$-induced collagen gel contraction (Figure 5f).

Importantly, LDE225 (10 or $15 \mu \mathrm{M})$ treatment of ex vivo human liver slices resulted in a significant inhibition of fibrotic genes (Acta2, TIMP-1 and PDGF $\beta$ R) (Figure 5g) and attenuation of the expression of ITGA11 and ITGA5 (angiogenesis parameters) and hedgehog signaling molecules Sox9 and Gli1 (Figure 5h). Altogether, these results confirm a potential link between ITGA11 and the hedgehog pathway.
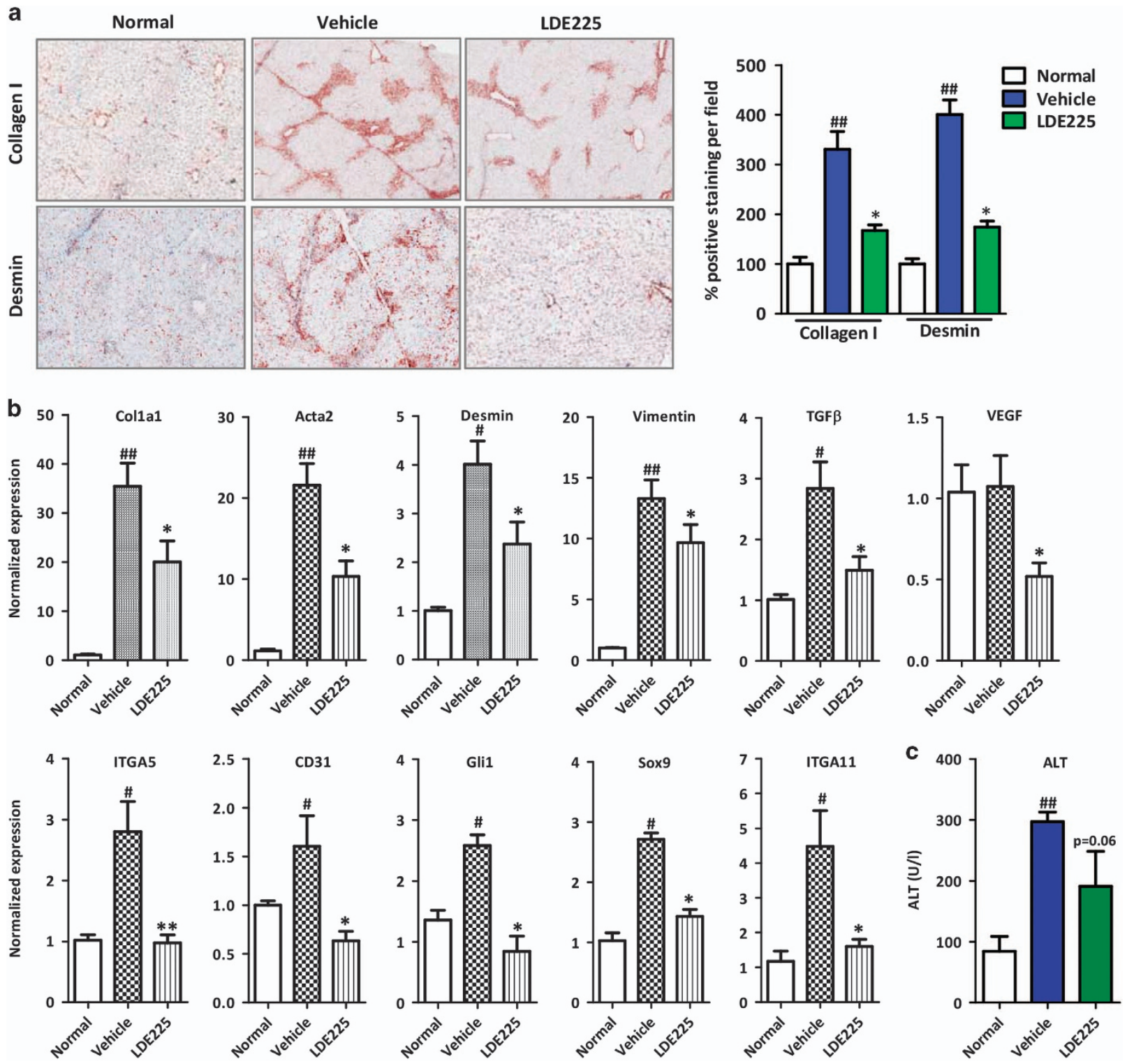

Figure 6 Effect of hedgehog inhibition in an acute $\mathrm{CCl}_{4}$-induced liver injury mouse model. (a) Representative photomicrographs and quantitative analysis of collagen-I- and desmin-stained liver sections from normal (olive-oil-treated), vehicle-treated CCl ${ }_{4}$ and $\mathrm{LDE} 225$ treated $\mathrm{CCl}_{4}$ mice. (b) Gene expression in the livers of different treated groups, $n=5$ per group. ${ }^{\#} P<0.05$ and ${ }^{\# \#} P<0.01$ versus the olive-oil-treated normal group; ${ }^{*} P<0.05$ versus $\mathrm{CCl}_{4}$-treated vehicle group. (c) The serum ALT levels of different treated groups. $n=5$ per group. ${ }^{\# \#} P<0.01$ versus olive-oil treated control group; $P=0.06$ versus $\mathrm{CCl}_{4}$-treated vehicle group. 
Inhibition of the hedgehog pathway ameliorates fibrogenesis in vivo in an acute liver injury mouse model

We further investigated the effect of a selective hedgehog signaling pathway inhibitor, LDE225 (or Erismodegib), in vivo in an acute liver fibrogenesis mouse model. In the $\mathrm{CCl}_{4}$-induced acute liver injury mouse model, hedgehog inhibitor significantly inhibited collagen-I, desmin and $\alpha$-SMA protein expression (Figure $6 a$ and Supplementary Figure S5). In addition, we observed a significant reduction in the mRNA expression of (a) fibrogenic parameters (Colla1, Acta2, Desmin, Vimentin and Tgf $\beta$ ), (b) angiogenesis markers (Vegf, Itga5 and Cd31) and (c) hedgehog pathway-related genes (Gli1 and Sox9) (Figure 6b) with significantly reduced ITGA11 expression (Figure 6b). We also observed a reduction $(P=0.06)$ in alanine aminotransferase levels following treatment with LDE225, suggesting the attenuation of liver inflammation (Figure 6c).

\section{Myofibroblast-specific localization of ITGA11 in mouse and human fibrotic kidneys}

Because renal myofibroblasts are the major mediator of ECM deposition and scar tissue formation in kidney fibrosis, we examined the role of ITGA11 in human fibrotic kidneys and UUO kidney fibrosis mouse models. After 7 days of UUO, mice developed extensive renal fibrosis as shown by the substantial deposition of collagen-I and enhanced $\alpha$-SMA expression in the fibrotic kidneys compared with that in the contralateral control kidneys (Figure 7a). There was strong expression of ITGA11 in the UUO kidneys in the tubulointerstitium, glomerular and perivascular membrane, the areas where $\alpha$-SMA was also highly expressed. By contrast, ITGA11 was weakly expressed in the glomerular mesangium in the contralateral kidneys (Figure 7a). Furthermore, in the fibrotic kidneys from UUO (3 and 7 days), we observed increased mRNA expression of Colla1, Acta2, Col3a1 and Itgal1 (Figure 7a). In human fibrotic kidneys, there was a significant increase in ITGA11 expression, which was specifically co-localized with $\alpha$-SMA-positive cells, including vascular smooth muscle cells and interstitial fibroblasts (Figure $7 \mathrm{~b}$ ). We also correlated the interstitial fibrosis and tubular atrophy score with ITGA11 expression using clinical transcriptomic data analysis (GEO accession number: GSE25902) and found that ITGA11 expression was significantly $(P<0.03)$ induced at score 3 compared with that at scores $0-2$ (Figure $7 \mathrm{c}$ ).

\section{Myofibroblast-specific localization of ITGA11 in the human fibrotic lungs}

To further extend our findings, we analyzed the ITGA11 expression in fibrotic lungs from patients with idiopathic pulmonary fibrosis. We found a significant induction in the ITGA11 expression in the fibrotic lungs as compared with the healthy lungs, and ITGA11 expression was found to be specifically co-localized with $\alpha$-SMA-positive myofibroblasts (Figure 7d). Furthermore, we found a highly significant increase in ITGA11 mRNA expression in the lungs from idiopathic pulmonary fibrosis patients $(P<0.001, n=123)$ as compared with normal lungs $(n=96)$, as assessed by transcriptome data analysis (GEO accession number: GSE47460) (Figure 7e). In addition, we assessed ITGA11 expression in normal and fibrotic human lung slices and found an increased expression of fibrotic parameters (Collagen-I, Vimentin and TIMP1) with concomitant induction in the ITGA11 expression (Figure 7f).

\section{DISCUSSION}

The present study established the significance of integrin alpha11 (ITGA11), a collagen I binding receptor, in the regulation of myofibroblast phenotypic differentiation during fibrotic diseases. ITGA11 expression was substantially induced in myofibroblasts in the fibrotic liver, kidneys and lungs and co-localized with $\alpha$-SMA in mouse models and human patient samples. Interestingly, the knockdown of ITGA11 in myofibroblasts (or activated HSCs) strongly inhibited their activation, differentiation, migration and contractility. Gene array showed that several fibrosis-related genes were significantly reduced after knockdown of ITGA11 in HSCs. Furthermore, our data revealed that the hedgehog signaling pathway contributed to the regulation of ITGA11 expression, and inhibition of the hedgehog pathway using a specific inhibitor attenuated early liver fibrogenesis in mice and inhibited fibrotic parameters in ex vivo human liver slices.

Excessive tissue scarring or fibrosis is a common feature of most chronic diseases. ${ }^{15}$ During tissue injury, environmental cues and various growth factors lead to the activation and differentiation of myofibroblasts, which are the major ECMproducing fibrogenic cells. ${ }^{12}$ Therefore, therapeutic intervention leading to inactivation of the myofibroblastic phenotype or reverting these cells to the quiescent state would be a rational approach to treat fibrotic diseases. Integrins are overexpressed in different cell types in response to an injury and interact with ECM proteins, allowing the cells to attach, migrate and proliferate. ${ }^{16}$ During fibrogenesis, myofibroblasts produce ECM constituents, including collagens, and express integrin receptors that interact and signal via ECM proteins. Recently, integrin $\alpha \mathrm{v}$ has been shown to regulate myofibroblast differentiation. ${ }^{17,20}$ As shown in the current study, ITGA11 was highly upregulated in myofibroblasts during fibrogenesis (liver, kidney and pulmonary fibrosis as shown in mouse models and human fibrotic tissues). Furthermore, negligible expression of ITGA11 was found in healthy mouse and human tissues, suggesting that the inhibition of this receptor likely has a minimal impact on normal tissues. Strikingly, the patient transcriptomic data analysis showed a significant induction of ITGA11 at increasing stages of fibrosis in patients with nonalcoholic fatty liver disease, idiopathic pulmonary fibrosis and interstitial kidney fibrosis. Furthermore, our gene expression data in freshly isolated lung specimens from patients showed higher ITGA11 expression levels in fibrotic lungs than those in non-fibrotic lungs. These data strongly highlight ITGA11 as a promising marker in fibrogenesis that could be used to identify and segregate fibrosis stages in different organs. 
a
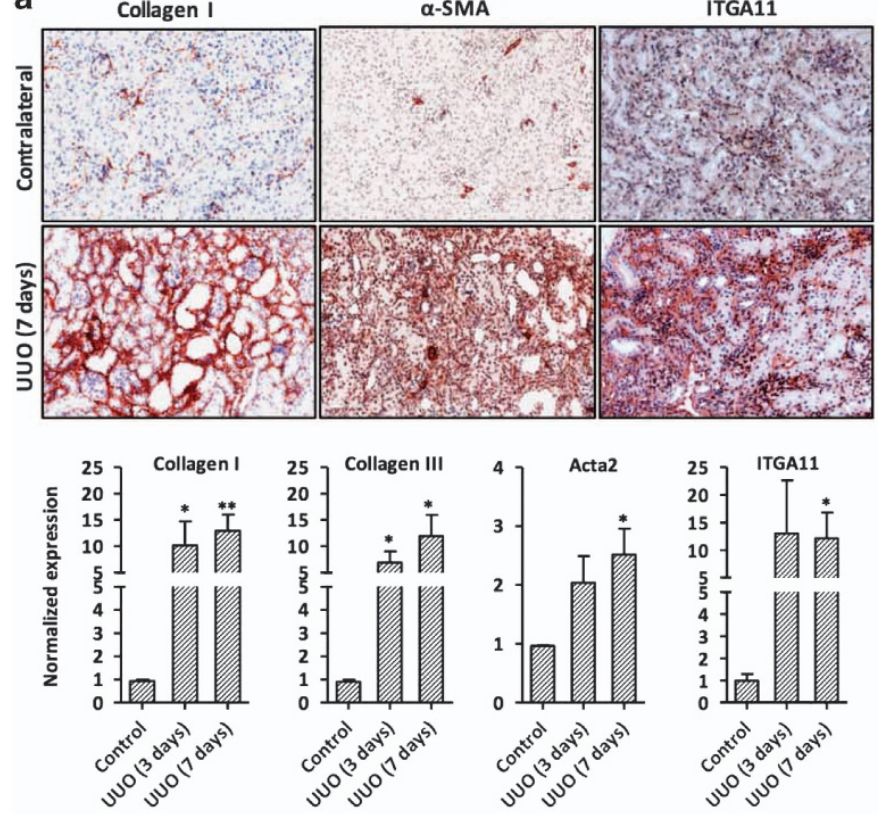

d
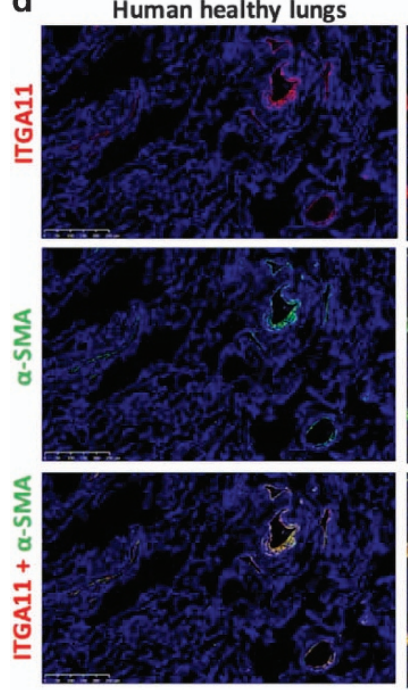
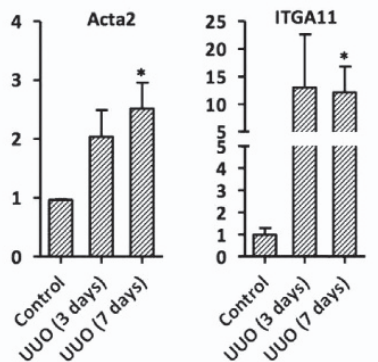
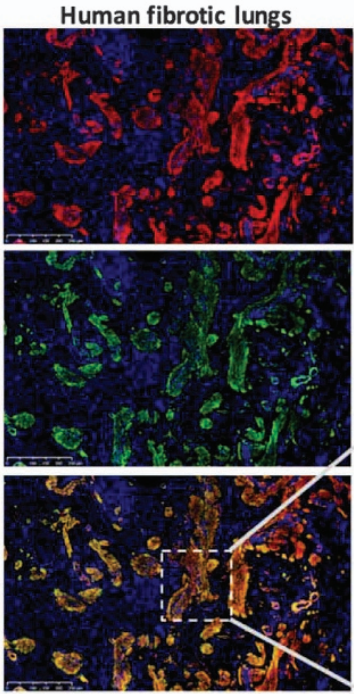

b
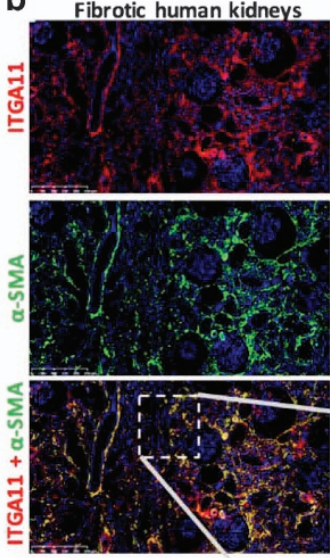

C
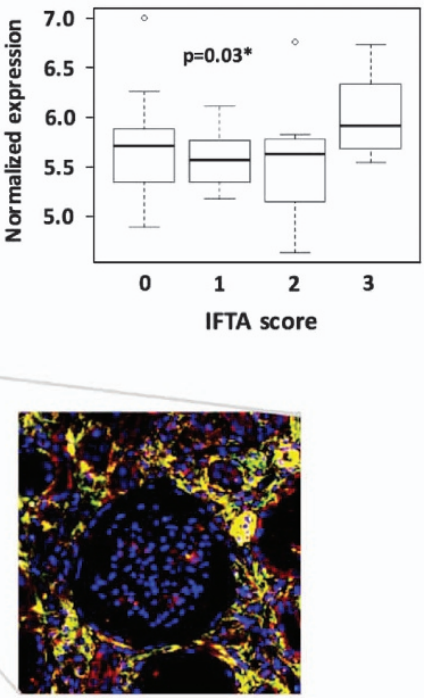

f

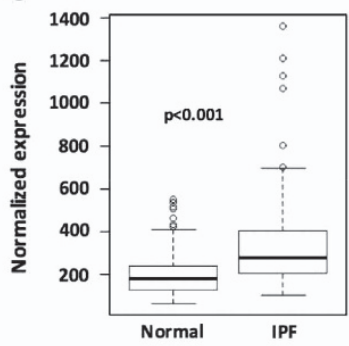

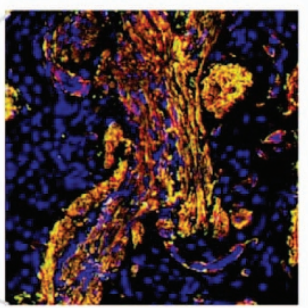

Figure 7 Integrin alpha 11 (ITGA11) overexpression in the UUO model of kidney fibrosis in mice and in fibrotic human kidneys and fibrotic lung tissues. (a) Collagen-I-, $\alpha$-SMA- and ITGA11-stained kidney sections obtained from sham- and 7 days UUO-operated mice ( $n=4$ per group). Gene expression of fibrotic parameters (Col1A1, Acta2 and Col3A1) and ITGA11 in the kidneys from contralateral, 3-day and 7-day UUO-operated mice, $n=4$ per group. ${ }^{*} P<0.05$ and $* * P<0.01$ versus contralateral kidneys. (b) $\alpha$-SMA and ITGA11 co-immunostained human fibrotic kidney sections ( $n=4$ per group). Upper, ITGA11 (red); middle, $\alpha$-SMA (green); bottom, merged image. Nuclei are stained blue using DAPI. The lower panel depicts the magnified image. (c) The ITGA11 mRNA expression levels extracted from publicly available transcriptome profiling data sets (www.ncbi.nIm.nih.gov/geo). Kidney tissues stratified according to the IFTA score: 0, no fibrosis $(n=16)$; 1 , mild fibrosis $(n=11) ; 2$, moderate fibrosis $(n=13)$; 3 , severe fibrosis $(n=8)(\mathrm{GSE} 25902)$, * $P=0.03$. (d) $\alpha-\mathrm{SMA}$ and ITGA11 co-immunostained human lung sections ( $n=5$ per group). Upper, ITGA11 (red); middle, $\alpha$-SMA (green); bottom, merged image. Nuclei are stained blue using DAPI. (e) The ITGA11 mRNA expression levels were extracted from publicly available transcriptome profiling data sets (www.ncbi.nlm.nih.gov/geo). Lung tissues with idiopathic pulmonary fibrosis $(n=123)$ compared with normal controls ( $n=96)$ (GSE47460). ${ }^{*} P<0.001$ versus normal lungs. (f) Heat map representation of the gene expression of ITGA11, Col1A1, Vimentin and TIMP1 in human lung slices. N1-N5 and F1-F5 denote normal and fibrotic samples, respectively.

During fibrogenesis, TGF $\beta$ is one of the key growth factor involved in disease progression by activated fibroblasts. ${ }^{11,33}$ TGF $\beta$ can increase the expression of integrins, ${ }^{34}$ and similarly, integrins can modulate the TGF $\beta /$ Smad pathways directly or indirectly via different mechanisms. ${ }^{17}$ In the current study,
ITGA11 expression in HSCs was highly upregulated in response to TGF $\beta$ activation (Figures $2 \mathrm{a}-\mathrm{c}$ ), corroborating with the previous findings showing TGF $\beta$-mediated regulation of ITGA11 in Smad- and Sp1-dependent manner. ${ }^{27}$ Furthermore, stable knockdown of ITGA11 in HSCs downregulated TGF $\beta$ 
superfamily genes (CEBPB, TGFBR1, TGFBR2 and TGIF1), demonstrating a strong negative feedback regulation of the TGF $\beta$ signaling pathway by ITGA11 in HSCs. In addition, fibrosis profiler array data revealed that ITGA11 knockdown in HSCs also reduced several pro-fibrotic and ECM remodeling genes (for example, collagen-I, MMP-1, $-3,-9$ and TIMP-1, -3), suggesting that ITGA11 controls ECM production and remodeling in HSCs. Integrins are also crucial receptors for the maintenance of the cytoskeleton, thereby controlling cell shape, contractility and migration. In the present study, we demonstrate that ITGA11 knockdown in HSCs led to a significant loss of focal adhesion, as shown with AFM, and a reduction in a major adhesion protein, paxillin, which regulates HSC adhesion to the matrix. As focal adhesions are crucial for cell contractility and migration, ITGA11 knockdown in HSCs led to reduced collagen gel contraction and migration.

Recent data have suggested that myofibroblasts in different fibrotic diseases originate from Gli1+ perivascular cells. ${ }^{32}$ Here we also demonstrate Gli1 co-expression on perivascular $\alpha$ SMApositive myofibroblasts in human fibrotic livers. Glil is a transcription factor of the hedgehog signaling pathway that has an important role in fibrosis, and its selective inhibition has been shown to ameliorate liver fibrosis. ${ }^{35}$ In this study, hedgehog signaling pathway molecules (Gli1 and Sox9) were upregulated in both TGF $\beta$-activated HSCs and in vivo in mouse liver fibrosis model. Interestingly, hedgehog pathway-related genes (Shh, Ptch1, Smo, Gli1, Gli2 and Sox9) were significantly downregulated in ITGA11-KD HSCs, and TGF $\beta$-induced upregulation of hedgehog signaling molecules (Sox9 and Gli1) were attenuated in ITGA11 knockdown HSCs, indicating an inter-relationship between the ITGA11 and hedgehog signaling pathways. We further observed significantly increased expression of ITGA11 in Shh-treated control HSCs that was completely blocked in ITGA11-KD cells, suggesting a direct correlation between the hedgehog pathway and ITGA11. Furthermore, treatment with a specific hedgehog inhibitor, LDE225, reduced the expression of ITGA11 and several fibrosis-related genes in TGF $\beta$-activated human HSCs and $\mathrm{CCl}_{4}$-induced early liver fibrogenesis mouse model. To demonstrate the impact of hedgehog inhibition on ITGA11 in clinical samples, we used precision-cut liver slices from patient-derived fibrotic livers. ${ }^{36}$ Interestingly, LDE225 strongly reduced the ITGA11 expression and several fibrotic genes in these slices. These data explicitly show that the ITGA11 and hedgehog pathways regulate each other, and the inhibition of the hedgehog pathway is an interesting strategy to inhibit the ITGA11 expression. Because ITGA11 positively regulates the TGF $\beta$ and hedgehog signaling pathways, ITGA11 might be involved downstream in these signaling pathways. However, detailed promoter studies are required to elucidate the role of the TGF $\beta /$ smad3 pathway and hedgehog/Gli1 pathway and involvement of ITGA11 in these regulatory pathways.

In conclusion, ITGA11 is a highly promising target that is selectively overexpressed in myofibroblasts during fibrotic diseases and regulates myofibroblast differentiation and key phenotypic characteristics. Furthermore, our data uncovered the hedgehog signaling pathway as a mediator of the ITGA11 pathway in myofibroblasts in vivo and in patient fibrotic tissues ex vivo. Collectively, these data highlight the potential therapeutic significance of ITGA11 in liver fibrosis and suggest that the development of strategies to antagonize ITGA11 could lead to the development of novel and effective therapies against fibrotic diseases.

\section{CONFLICT OF INTEREST}

JP is the founder and stakeholder of ScarTec Therapeutics BV, Enschede, The Netherlands. The other authors declare no conflict of interest.

\section{ACKNOWLEDGEMENTS}

This project was supported by the Netherlands Organization for Health Research and Development (ZonMW, NWO)-funded VENI innovation grant 916.151.94 (to RB), Swedish Research Council grant (Project number: 2011-5389, to JP), Innovation grant from the Dutch Kidney Foundation (14OI16; to JP), Irma T. Hirschl Trust funding and NIH/NIDDK (DK099558) (to YH) and NIH funding DK56621 (to SLF).

Author contributions: RB and JP conceived and designed the project. $\mathrm{RB}$ performed the experiments. W-MS and APK performed informatics analysis. SY performed the UUO mouse model. SN performed the human lung and liver slice experiments. SN, JVB, CAP, HW, NK, AV and SY contributed the materials. RB and JP analyzed the data and wrote the manuscript. YH, HVG, GS and SLF read and critically revised the manuscript.

\section{PUBLISHER'S NOTE}

Springer Nature remains neutral with regard to jurisdictional claims in published maps and institutional affiliations.

1 Wynn TA. Cellular and molecular mechanisms of fibrosis. J Pathol 2008; 214: 199-210.

2 Desmouliere A, Darby IA, Gabbiani G. Normal and pathologic soft tissue remodeling: role of the myofibroblast, with special emphasis on liver and kidney fibrosis. Lab Invest 2003; 83: 1689-1707.

3 Hinz B, Phan SH, Thannickal VJ, Prunotto M, Desmouliere A, Varga J et al. Recent developments in myofibroblast biology: paradigms for connective tissue remodeling. Am J Pathol 2012; 180: 1340-1355.

4 Sugimoto H, Mundel TM, Kieran MW, Kalluri R. Identification of fibroblast heterogeneity in the tumor microenvironment. Cancer Biol Ther 2006; 5: 1640-1646.

5 Wynn TA. Integrating mechanisms of pulmonary fibrosis. J Exp Med 2011; 208: 1339-1350

6 Hinz B, Phan SH, Thannickal VJ, Galli A, Bochaton-Piallat ML, Gabbiani G. The myofibroblast: one function, multiple origins. Am J Pathol 2007; 170: 1807-1816.

7 Humphreys BD, Lin SL, Kobayashi A, Hudson TE, Nowlin BT, Bonventre JV et al. Fate tracing reveals the pericyte and not epithelial origin of myofibroblasts in kidney fibrosis. Am J Pathol 2010; 176: 85-97.

8 Kalluri R, Neilson EG. Epithelial-mesenchymal transition and its implications for fibrosis. J Clin Invest 2003; 112: 1776-1784.

9 Thiery JP, Acloque H, Huang RY, Nieto MA. Epithelial-mesenchymal transitions in development and disease. Cell 2009; 139: 871-890.

10 Piera-Velazquez S, Li Z, Jimenez SA. Role of endothelial-mesenchymal transition (EndoMT) in the pathogenesis of fibrotic disorders. Am J Pathol 2011; 179: 1074-1080.

11 Friedman SL. Mechanisms of hepatic fibrogenesis. Gastroenterology 2008; 134: 1655-1669.

12 Gabbiani G. The myofibroblast in wound healing and fibrocontractive diseases. J Pathol 2003; 200: 500-503. 
13 Gressner OA, Weiskirchen R, Gressner AM. Evolving concepts of liver fibrogenesis provide new diagnostic and therapeutic options. Comp Hepatol 2007; 6: 7 .

14 Trautwein C, Friedman SL, Schuppan D, Pinzani M. Hepatic fibrosis: concept to treatment. J Hepatol 2015; 62: S15-S24.

15 Wynn TA, Ramalingam TR. Mechanisms of fibrosis: therapeutic translation for fibrotic disease. Nat Med 2012; 18: 1028-1040.

16 Agarwal SK. Integrins and cadherins as therapeutic targets in fibrosis. Front Pharmacol 2014; 5: 131.

17 Henderson NC, Sheppard D. Integrin-mediated regulation of TGFbeta in fibrosis. Biochim Biophys Acta 2013; 1832: 891-896.

18 Humphries JD, Byron A, Humphries MJ. Integrin ligands at a glance. J Cell Sci 2006; 119: 3901-3903.

19 Hynes RO. Integrins: bidirectional, allosteric signaling machines. Cell 2002; 110: 673-687.

20 Henderson NC, Arnold TD, Katamura Y, Giacomini MM, Rodriguez JD, McCarty $\mathrm{JH}$ et al. Targeting of alphav integrin identifies a core molecular pathway that regulates fibrosis in several organs. Nat Med 2013; 19: 1617-1624.

21 Munger JS, Huang X, Kawakatsu H, Griffiths MJ, Dalton SL, Wu J et al. The integrin alpha $v$ beta 6 binds and activates latent TGF beta 1: a mechanism for regulating pulmonary inflammation and fibrosis. Cell 1999; 96: 319-328.

22 Popova SN, Lundgren-Akerlund E, Wiig H, Gullberg D. Physiology and pathology of collagen receptors. Acta Physiol (Oxf) 2007; 190: 179-187.

23 Tiger CF, Fougerousse F, Grundstrom G, Velling T, Gullberg D. $\alpha 11 \beta 1$ integrin is a receptor for interstitial collagens involved in cell migration and collagen reorganization on mesenchymal nonmuscle cells. Dev Biol 2001; 237: 116-129.

24 Popova SN, Rodriguez-Sanchez B, Liden A, Betsholtz C, Van Den Bos T, Gullberg D. The mesenchymal alpha11 beta1 integrin attenuates PDGF-BBstimulated chemotaxis of embryonic fibroblasts on collagens. Dev Biol 2004; 270: 427-442.

25 Talior-Volodarsky I, Connelly KA, Arora PD, Gullberg D, McCulloch CA. $\alpha 11$ integrin stimulates myofibroblast differentiation in diabetic cardiomyopathy. Cardiovasc Res 2012; 96: 265-275.

26 Honda E, Yoshida K, Munakata H. Transforming growth factor-beta upregulates the expression of integrin and related proteins in MRC-5 human myofibroblasts. Tohoku J Exp Med 2010; 220: 319-327.

27 Lu N, Carracedo S, Ranta J, Heuchel R, Soininen R, Gullberg D. The human alpha11 integrin promoter drives fibroblast-restricted expression

in vivo and is regulated by TGF-beta1 in a Smad- and Sp1dependent manner. Matrix Biol 2010; 29: 166-176.

28 Carracedo S, Lu N, Popova SN, Jonsson R, Eckes B, Gullberg D. The fibroblast integrin alpha11beta1 is induced in a mechanosensitive manner involving activin $\mathrm{A}$ and regulates myofibroblast differentiation. J Biol Chem 2010; 285: 10434-10445.

29 Navab R, Strumpf D, To C, Pasko E, Kim KS, Park CJ et al. Integrin alpha1 1 beta1 regulates cancer stromal stiffness and promotes tumorigenicity and metastasis in non-small cell lung cancer. Oncogene 2016; 35: 1899-1908.

30 Vaira V, Fedele G, Pyne S, Fasoli E, Zadra G, Bailey D et al. Preclinical model of organotypic culture for pharmacodynamic profiling of human tumors. Proc Natl Acad Sci USA 2010; 107: 8352-8356.

31 Intraobserver and interobserver variations in liver biopsy interpretation in patients with chronic hepatitis $\mathrm{C}$. The French METAVIR Cooperative Study Group. Hepatology 1994; 20: 15-20.

32 Kramann R, Schneider RK, DiRocco DP, Machado F, Fleig S, Bondzie PA et al. Perivascular Gli1+ progenitors are key contributors to injury-induced organ fibrosis. Cell Stem Cell 2015; 16: 51-66.

33 Inagaki Y, Okazaki I. Emerging insights into transforming growth factor beta Smad signal in hepatic fibrogenesis. Gut 2007; 56: 284-292.

34 Margadant C, Sonnenberg A. Integrin-TGF-beta crosstalk in fibrosis, cancer and wound healing. EMBO Rep 2010; 11: 97-105.

$35 \mathrm{Hu} \mathrm{L}$, Lin X, Lu H, Chen B, Bai Y. An overview of hedgehog signaling in fibrosis. Mol Pharmacol 2015; 87: 174-182.

36 Olinga $P$, Schuppan D. Precision-cut liver slices: a tool to model the liver ex vivo. J Hepatol 2013; 58: 1252-1253.

cc) (i) (5)

This work is licensed under a Creative Commons

Attribution-NonCommercial-ShareAlike

International License. The images or other third party material in this article are included in the article's Creative Commons license, unless indicated otherwise in the credit line; if the material is not included under the Creative Commons license, users will need to obtain permission from the license holder to reproduce the material. To view a copy of this license, visit http:// creativecommons.org/licenses/by-nc-sa/4.0/

(C) The Author(s) 2017

Supplementary Information accompanies the paper on Experimental \& Molecular Medicine website (http://www.nature.com/emm) 